\title{
Positive periodic solutions for impulsive differential equations with infinite delay and applications to integro-differential equations
}

\author{
Sebastián Buedo-Fernández \\ Departamento de Estatística, Análise Matemática e Optimización and Instituto de Matemáticas, \\ Facultade de Matemáticas, Universidade de Santiago de Compostela, Campus Vida, 15782 \\ Santiago de Compostela, Spain \\ Teresa Faria ${ }^{1}$ \\ Departamento de Matemática and CMAF-CIO, Faculdade de Ciências, \\ Universidade de Lisboa, Campo Grande, 1749-016 Lisboa, Portugal
}

\begin{abstract}
Sufficient conditions for the existence of at least one positive periodic solution are established for a family of scalar periodic differential equations with infinite delay and nonlinear impulses. Our criteria, obtained by applying a fixed point argument to an original operator constructed here, allow to treat equations incorporating a rather general nonlinearity and impulses whose signs may vary. Applications to some classes of Volterra integro-differential equations with unbounded or periodic delay and nonlinear impulses are given, extending and improving results in the literature.
\end{abstract}

Keywords: delay differential equations, impulses, infinite delay, positive periodic solution, fixed point theorems in cones, Volterra integro-differential equations.

2010 Mathematics Subject Classification: 34K13, 34K45, 92D25

\section{Introduction}

In recent years, much attention has been dedicated to the study of differential equations with delays and impulses, since they often produce realistic models for evolutionary systems which go through abrupt changes, caused by random or predictable external factors. In the case of autonomous equations, it is particular relevant to study the existence and attractivity of equilibria. Periodic phenomena however contribute significantly in population dynamical systems, artificial neural networks and many other biological and physical processes as well, in which case the models are better portrayed by periodic differential equations. For periodic differential equations, without and with impulses, a key question is whether there exists any periodic solution, which in many models, due to their real world interpretation, is required to be positive.

In this paper, we consider a family of periodic scalar differential equations with infinite delay and impulses, written in the general abstract form

$$
\begin{cases}x^{\prime}(t)=-a(t) x(t)+g\left(t, x_{t}\right), & t \geq 0, t \neq t_{k}, k \in \mathbb{N} \\ x\left(t_{k}^{+}\right)-x\left(t_{k}\right)=I_{k}\left(x\left(t_{k}\right)\right), & k \in \mathbb{N}\end{cases}
$$

\footnotetext{
${ }^{1}$ Corresponding author. E-mail: teresa.faria@fc.ul.pt
} 
where the scalar functions $a(t)$ and $g(t, \varphi)$ are continuous, nonnegative and periodic in $t$ with a common period $\omega>0$, the impulsive functions $I_{k}(u)$ are continuous, $k \in \mathbb{N}$, and the impulses $\Delta x\left(t_{k}\right):=x\left(t_{k}^{+}\right)-x\left(t_{k}\right)$ occur at times $t_{k} \nearrow \infty$ with a periodicity $\omega$ to be specified in the next section. Here, $g:[0, \infty) \times D \rightarrow \mathbb{R}$, where $D \subset \mathcal{B}$ and $\mathcal{B}$ is an adequate Banach space of piecewise continuous functions defined on $(-\infty, 0]$ with values in $\mathbb{R}$, which are left-continuous. As usual, $x_{t}$ denotes the entire past history of the system up to time $t$, or in other words, $x_{t}(s)=x(t+s)$ for $s \leq 0$. Eq. (1.1) sets a general framework for many relevant models from mathematical biology and other sciences, for which only positive (or nonnegative) solutions of (1.1) are meaningful.

The main aim of the paper is to establish sufficient conditions for the existence of positive periodic solutions for (1.1). Our technique is based on a version of the Krasnoselskii fixed point theorem in cones [16] due to Guo [11], which is applied to a convenient operator whose fixed points are precisely the $\omega$-periodic solutions of (1.1) we are looking for.

In fact, there is an extensive literature on the application of fixed point theorems, such as Banach contraction principle, Schauder or Krasnoselskii theorems, the continuation theorem, as well as other methods, which include lower and upper solutions, monotone iterative schemes, combinations with Lyapunov functionals - to the quest of positive periodic solutions to delay differential equations (DDEs). Related to our research, we refer to the early works of Nieto [22], Chen [4], Jiang and Wei [15], and to $[2,10,19,20,21,26,27,28,31,34,35]$, most of them considering DDEs with a single discrete delay. See also $[6,36]$, for results about permanence implying existence of positive periodic solutions for DDEs. In spite of this wide array of techniques from nonlinear analysis, our method is essentially new, since it relies on a fixed point argument applied to an original operator constructed here. Our method, applicable under very mild restrictions, improves previous results in the literature, and has straightforward extensions to other classes of impulsive DDEs.

The work reported here was highly motivated by the papers $[9,15,19]$, which we refer to also for further references. In [19], and extending the ideas in [15, 22], Li et al. considered a family of impulsive DDEs, with a single discrete delay and positive impulses:

$$
\begin{cases}x^{\prime}(t)=-a(t) x(t)+f(t, x(t-\tau(t))), & t \geq 0, t \neq t_{k}, k \in \mathbb{N} \\ x\left(t_{k}^{+}\right)-x\left(t_{k}\right)=I_{k}\left(x\left(t_{k}\right)\right), & k \in \mathbb{N}\end{cases}
$$

with $a(t), f(t, u), \tau(t)$ continuous, nonnegative and $\omega$-periodic in $t,\left(t_{k}\right),\left(I_{k}\right)$ as in (1.1), and moreover with $I_{k}(u)>0$ for $u>0, k \in \mathbb{N}$. As in [21,30,34, 35] and many other papers, in [19] the authors found positive periodic solutions as fixed points for a certain operator via a Krasnoselskii fixed point theorem; since the definition of such operator requires summing all the impulses (multiplied by a Green function) up to time $t$, the impulses must be positive in order to be sure that the operator maps a cone of positive functions into itself. This creates some difficulties in applications, and it turns out that some results in [19] cannot be used in the simple case of positive linear impulses (i.e., when $I_{k}(u)=b_{k} u$ with $\left.b_{k}>0, k \in \mathbb{N}\right)$. On the other hand, from the point of view of applications it is very restrictive to consider only positive impulses. For a discussion of the role of impulses, see e.g. $[20,25]$.

Recently, by introducing a different operator, Faria and Oliveira [9] studied more general equations with either finite multiple discrete delays or finite distributed delay - however, due to technical 
difficulties, only linear impulses were considered, as follows:

$$
\left\{\begin{array}{l}
x^{\prime}(t)+a(t) x(t)=g\left(t, x_{t}\right), \quad t_{0} \leq t \neq t_{k} \\
x\left(t_{k}^{+}\right)-x\left(t_{k}\right)=b_{k} x\left(t_{k}\right), \quad k \in \mathbb{N}
\end{array}\right.
$$

with $a(t), g(t, \varphi),\left(t_{k}\right)$ as in $(1.1)$ and constants $b_{k} \in(-1, \infty)$. The existence of positive periodic solutions for periodic DDEs (1.1) with impulses given by general continuous functions $I_{k}(u)$ which may change sign was the principal motivation for this paper, which can be seen as a continuation of the work in [9]. We emphasise however that, even for impulsive DDEs (1.3) with finite delay, the results in this paper are more general than the ones in [9].

One should stress that DDEs with infinite delay require a rigorous abstract formulation in an admissible phase space $[13,14]$, in order to guarantee that the initial value problems are well-posed and that the standard qualitative properties of solutions are valid.

The contents and organization of the next sections are now described. Section 2 is a section of preliminaries, and begins with the choice of an appropriate Banach phase space to treat general equations with infinite delays and impulses. Afterwards, we set the main hypotheses for (1.1), and define a suitable cone $K$ and a suitable operator $\Phi$ on $K \backslash\{0\}$, whose fixed points are precisely the positive $\omega$-periodic solutions to (1.1).

Section 3 contains the main results of the paper, which establish sufficient conditions for the existence of fixed points of $\Phi$. Such conditions arise by analysing the interplay between the behaviour of the impulse functions and of the nonlinear terms, for solutions next to zero or to infinity, and are easily verified in practice. The Krasnoselskii-Guo theorem [11] is used, both in its compressive and expansive forms. Several criteria based on either a pointwise or an average comparison of $a(t)$ and $g(t, \varphi)$ for $t \in[0, \omega]$ and $\|\varphi\|$ in the vicinity of zero or infinity, are derived. Our results are illustrated and analysed within the context of some related literature.

In Section 4, applications to impulsive Volterra integro-differential equations with delay are given, with emphasis on the case of unbounded distributed delay. We remark that the situation with infinite delay has not often been addressed, the works of Zhao [36] and Jiang and Wei [15] for DDEs without impulses being exceptions. Generalisations of results in [15] are given in Subsection 4.1, namely we highlight the treatment of a mixed monotonicity model. The case of impulsive DDEs with bounded periodic distributed delay is analysed in Subsection 4.2, extending and enhancing previous criteria in e.g. [2]. The models in these subsections are broad enough to include generalised Nicholson's blowflies and Mackey-Glass equations as particular concrete examples. Some attention to impulses satisfying the requirements set in Section 2 is given in Subsection 4.3. We restrict ourselves only to few applications and selected examples, to reduce the size of this manuscript; many other examples can be given, e.g. straightforward generalisations of the models treated in $[2,9,19,34]$. A short section of final comments ends the paper.

\section{An abstract framework and preliminary results}

We now set an appropriate phase space to treat impulsive differential equations with infinite delay. Although this abstract formulation does not seem to be relevant for the strict purpose of finding periodic solutions of (1.1), it is essential to consider equations with abstract nonlinear terms $g\left(t, x_{t}\right)$, instead of the form $g(t, x(t-\tau(t)))$, or even $g\left(t, \int_{-\tau(t)}^{0} k(s) x(t+s) d s\right)$ for some $g: \mathbb{R}^{+} \times \mathbb{R} \rightarrow \mathbb{R}$. 
Moreover, for other reasons, e.g. to pursue with the study of their attractivity, it is important to realise that we shall look for periodic solutions inside a Banach space $X$ contained naturally in the phase space for (1.1). For more details and results of existence, uniqueness, global continuation of solutions for DDEs with impulses and infinite delay, we refer to [7, 24] and references therein. On the other hand, a reader mostly motivated by concrete applications may simply concentrate his/her attention on the definition of the space $X$ in (2.1).

For a real interval $I$, let $\mathcal{B}(I ; \mathbb{R})$ be the Banach space of bounded functions $\varphi: I \rightarrow \mathbb{R}$, endowed with the supremum norm $\|\cdot\|_{\infty}$. For a compact interval $I=[\alpha, \beta](\alpha<\beta)$, consider the subspace $P C(I ; \mathbb{R})$ of $\mathcal{B}(I ; \mathbb{R})$ of the piecewise continuous functions defined on $I$ which are left-continuous in $(\alpha, \beta]$, i.e., $P C(I ; \mathbb{R}):=\{\varphi: I \rightarrow \mathbb{R} \mid \varphi$ is continuous except for a finite number of elements of $I$ for which $\varphi\left(s^{-}\right)$and $\varphi\left(s^{+}\right)$exist and $\left.\varphi(s)=\varphi\left(s^{-}\right)\right\}$. The closure of $P C(I ; \mathbb{R})$ in $\mathcal{B}(I ; \mathbb{R})$ is the space $\mathcal{R}(I ; \mathbb{R})$ of normalized regulated functions on $I$.

Next we shall consider systems with "infinite memory", thus take $I=(-\infty, 0]$. Define $P C:=$ $P C((-\infty, 0] ; \mathbb{R})$ as the space of functions $\varphi:(-\infty, 0] \rightarrow \mathbb{R}$ whose restriction to any compact interval $[\alpha, \beta] \subset(-\infty, 0]$ is in $\mathcal{R}([\alpha, \beta] ; \mathbb{R})$. The elements of $P C$ are left-continuous and may have countable discontinuities of the first kind. Note however that $P C$ is not contained in $\mathcal{B}((-\infty, 0] ; \mathbb{R})$. We need to choose a subset of $P C$ and an appropriate norm, in such a way that the new space is a Banach space providing a suitable framework to handle DDEs with infinite delays and impulses [13, 14, 24]. We use a norm with a weight function $f$ satisfying the properties below:

(f1) $f:(-\infty, 0] \rightarrow[1, \infty)$ is a nonincreasing function with $f(0)=1$,

(f2) $\lim _{u \rightarrow 0^{-}} \frac{f(t+u)}{f(t)}=1$ uniformly on $(-\infty, 0]$,

(f3) $f(s) \rightarrow \infty$ as $s \rightarrow-\infty$.

The space

$$
P C_{f}:=\left\{\varphi \in P C((-\infty, 0] ; \mathbb{R}): \sup _{s \leq 0} \frac{|\varphi(s)|}{f(s)}<\infty\right\}
$$

is a Banach space (see [7, Lemma 3.1]) with the norm

$$
\|\varphi\|_{f}:=\sup _{s \leq 0} \frac{|\varphi(s)|}{f(s)} .
$$

Write $\mathbb{R}^{+}=[0, \infty), \mathbb{R}^{-}=(-\infty, 0]$. In the space $P C_{f}$, we consider a DDE with impulses in the abstract form (1.1), where $a: \mathbb{R}^{+} \rightarrow \mathbb{R}^{+}, g: \mathbb{R}^{+} \times P C_{f} \rightarrow \mathbb{R}^{+}\left(\right.$or $g: \mathbb{R}^{+} \times D \rightarrow \mathbb{R}^{+}$with $\left.D \subset P C_{f}\right), I_{k}: \mathbb{R}^{+} \rightarrow \mathbb{R}(k \in \mathbb{N})$ are continuous, and $\left(t_{k}\right)_{k \in \mathbb{N}}$ is an increasing sequence of positive real numbers, $t_{k} \rightarrow \infty$. As mentioned in the introduction, for any $b \in \mathbb{R}$ and $x:(-\infty, b) \rightarrow \mathbb{R}$ a piecewise continuous function, $x_{t}: \mathbb{R}^{-} \rightarrow \mathbb{R}$ for $t<b$ is defined by

$$
x_{t}(s)=x(t+s), \quad s \in \mathbb{R}^{-} .
$$

For non-impulsive versions of (1.1), we take as phase space the subset $C_{f}$ of continuous functions, $C_{f}=\left\{\varphi \in C((-\infty, 0] ; \mathbb{R}): \sup _{s \leq 0}(|\varphi(s)| / f(s))<\infty\right\}$, with the above norm $\|\cdot\|_{f}$. This space has often been considered in the literature of (non-impulsive) DDEs with infinite delay [14].

As usual, by a solution $x$ of $(1.1)$ on $[\sigma, b)$, with $0 \leq \sigma<b \leq \infty$, we mean a function $x:(-\infty, b) \rightarrow$ $\mathbb{R}$ such that $x_{t} \in P C_{f}$ for $t \in[\sigma, b), x, x^{\prime}$ are continuous on $[\sigma, b) \backslash\left\{t_{k}: k \in \mathbb{N}\right\}$, and satisfies (1.1). For 
(1.1), the subset of bounded functions $B P C:=P C((-\infty, 0] ; \mathbb{R}) \cap \mathcal{B}((-\infty, 0] ; \mathbb{R}) \subset P C_{f}$ is usually taken as the space of admissible initial conditions [7, 24].

We recall that a function $y: I \rightarrow \mathbb{R}$, with $I=\mathbb{R}, I=\mathbb{R}^{+}$or $I=\mathbb{R}^{-}$, is called $\omega$-periodic if $y(t+\omega)=y(t)$, for any $t, t+\omega \in I$. Since we are only interested in obtaining periodic solutions, the spaces $P C_{f}$ and $B P C$ are still too large for our purposes, hence we choose a subset of $P C_{f}$ that fits well with the nature of the solutions we are looking for.

Consider $\omega>0$ and points $t_{1}, \ldots, t_{p}$ (for some $p \in \mathbb{N}$ ) such that $0 \leq t_{1}<\cdots<t_{p}<\omega$, and define the sequence $\left(t_{k}\right)_{k \in \mathbb{N}}$ by $t_{k+n p}=t_{k}+n \omega$ for all $n \in \mathbb{Z}, k=1, \ldots, p$. Take $X$ as the space

$$
\begin{array}{r}
X=\left\{y: \mathbb{R} \rightarrow \mathbb{R} \mid y \text { is } \omega \text {-periodic, continuous for all } t \neq t_{k},\right. \\
\text { and } \left.y\left(t_{k}^{-}\right)=y\left(t_{k}\right), y\left(t_{k}^{+}\right) \in \mathbb{R}, \text { for } k \in \mathbb{Z}\right\},
\end{array}
$$

and

$$
\tilde{X}=\left\{y_{t}: y \in X, t \in \mathbb{R}\right\} .
$$

For $y \in X$ fixed, $y_{t} \in P C_{f}$ for all $t$, thus $\tilde{X} \subset P C_{f}$. In this way, with the identification $y \equiv y_{0}=$ $y_{(-\infty, 0]}, X$ can also be identified with a subspace of $\omega$-periodic functions in $P C_{f}$, and therefore seen as a (closed) subset of $P C_{f}$. We now take the supremum norm in $X$ and $\tilde{X},\|y\|_{\infty}=\sup _{t \in[0, \omega]}|y(t)|$.

Lemma 2.1. There exists $L>0$ such that, for any $y \in X$ and $t \in \mathbb{R},\left\|y_{t}\right\|_{f} \leq\|y\|_{\infty}=\left\|y_{t}\right\|_{\infty} \leq$ $L\left\|y_{t}\right\|_{f}$. In particular, the norms $\|\cdot\|_{f}$ and $\|\cdot\|_{\infty}$ are equivalent in both $\tilde{X}$ and $X$.

Proof. Let $y \in X, t \in \mathbb{R}$. One has $\|y\|_{\infty}=\left\|y_{t}\right\|_{\infty}$. On the one hand, we have $\left\|y_{t}\right\|_{f}=\sup _{s \leq 0} \frac{|y(t+s)|}{f(s)} \leq$ $\sup _{s \leq 0}|y(t+s)|=\sup _{s \in[0, \omega]}|y(s)|=\|y\|_{\infty}$. On the other hand, $\|y\|_{\infty}=\sup _{s \in[-\omega, 0]}|y(t+s)| \leq$ $L \sup _{s \leq 0} \frac{|y(t+s)|}{f(s)}=L\left\|y_{t}\right\|_{f}$, where $L:=\sup _{s \in[-\omega, 0]} f(s)=f(-\omega)$.

In view of the previous lemma, from now on we shall work in the Banach space $X$ with the norm $\|\cdot\|_{\infty}$, simply denoted by $\|\cdot\|$, thus $\mathbb{R}^{+} \times \tilde{X}$ is taken as the domain of $g(t, \varphi)$.

The following hypotheses on (1.1) will be assumed:

(A1) The functions $a: \mathbb{R}^{+} \rightarrow \mathbb{R}, g: \mathbb{R}^{+} \times \tilde{X} \rightarrow \mathbb{R}$ are nonnegative, continuous, not identically zero, $\omega$-periodic in $t \in \mathbb{R}^{+}$, for some $\omega>0$, and $g$ is bounded on bounded sets of $\mathbb{R}^{+} \times \tilde{X}$.

(A2) The functions $I_{k}:[0, \infty) \rightarrow \mathbb{R}$ are continuous and there is a positive integer $p$ such that $0 \leq t_{1}<\cdots<t_{p}<\omega$ and $t_{k+p}=t_{k}+\omega, I_{k+p}=I_{k}, k \in \mathbb{N}$.

(A3) There exist constants $a_{k}>-1$ and $b_{k}$ such that $a_{k} y \leq I_{k}(y) \leq b_{k} y$, for $y \geq 0, k \in\{1, \ldots, p\}$.

(A4) $\prod_{i=1}^{p}\left(1+b_{k}\right)<\exp \left(\int_{0}^{\omega} a(t) d t\right)$.

We give a few comments about the choice of the above hypotheses. Under (A2)-(A3), the constants $a_{k}$ and $b_{k}$ can be extended for $k>p$ by $\omega$-periodicity, $a_{p+k}=a_{k}, b_{k+p}=b_{k}, k \in \mathbb{N}$, and chosen as the sharpest ones: $a_{k}=\inf _{u>0} \frac{I_{k}(u)}{u}, b_{k}=\sup _{u>0} \frac{I_{k}(u)}{u}, k \in \mathbb{N}$. In [9], Faria and Oliveira studied impulsive periodic scalar equations (with finite delay) (1.3) subject to linear impulses $\Delta x\left(t_{k}\right):=x\left(t_{k}^{+}\right)-x\left(t_{k}\right)=b_{k} x\left(t_{k}\right)$, with $t_{k}$ and $I_{k}(u)=b_{k} u$ satisfying (A2). For such systems, it was imposed in [9] that $b_{k}>-1$, in order to guarantee that, after suffering an impulse at the 
instant $t_{k}$, a positive solution remains positive. Hypothesis (A3) above is a generalisation of such assumption. It also implies that $I_{k}(0)=0$ for all $k$, a constraint considered by many authors, see e.g. [29]. The situation $I_{k}(0) \neq 0$ will be analysed in a future work. Related to this aspect, see [23] and references therein, for possible extensions of our method to impulsive DDEs (1.1) for which $g(t, \varphi)$ has a singularity at $\varphi=0$. Condition (A4), also imposed in $[9,20]$, expresses that the impulses are not too large when compared with the average of $a(t)$ over an interval of length $\omega$. We remark that even in the case of linear impulses $I_{k}(u)=b_{k} u$, the stronger restriction $\prod_{i=1}^{p}\left(1+b_{k}\right)=1$ has often been imposed [28, 31, 33, 35], see further comments in [20].

Let $X^{+}$be the subset of $X$ of nonnegative functions, i.e., of functions $y \in X$ such that $y(t) \geq$ $0, t \in[0, \omega]$. In order to simplify the writing, we define the following auxiliary functions:

$$
\begin{aligned}
& A(t)=\int_{0}^{t} a(u) d u \quad \text { for } t \geq 0, \quad J_{k}(u)=\frac{u}{u+I_{k}(u)} \quad \text { for } u>0, k \in\{1, \ldots, p\}, \\
& B(t ; y)=\prod_{k: t_{k} \in[0, t)} J_{k}\left(y\left(t_{k}\right)\right) \quad \text { and } \\
& \tilde{B}(s, t ; y)=\frac{B(s ; y)}{B(t ; y)}=\prod_{k: t_{k} \in[t, s)} J_{k}\left(y\left(t_{k}\right)\right) \quad \text { for } 0 \leq t \leq s \leq t+\omega, y \in X^{+} \backslash\{0\} .
\end{aligned}
$$

Throughout the paper, we adopt the standard convention where a product is equal to one when the number of factors is zero.

From the definitions above and hypotheses (A2)-(A4), it is clear that

$$
\left(1+b_{k}\right)^{-1} \leq J_{k}(u) \leq\left(1+a_{k}\right)^{-1}, \quad u>0, k=1, \ldots, p .
$$

The function $\tilde{B}(s, t ; y)$ has the property $\tilde{B}(s+\omega, t+\omega ; y)=\tilde{B}(s, t ; y)$ for $0 \leq t \leq s \leq t+\omega, y \in$ $X^{+} \backslash\{0\}$. Since there is a finite number of impulses on each interval of length less than or equal to $\omega, \tilde{B}(s, t ; y)$ is uniformly bounded above and below by constants $\bar{B}, \underline{B} \in(0, \infty)$,

$$
\underline{B} \leq \tilde{B}(s, t ; y) \leq \bar{B} \quad \text { for } \quad 0 \leq t \leq s \leq t+\omega, y \in X^{+} \backslash\{0\},
$$

with $\bar{B} \leq \max \left\{\prod_{k=j}^{j+l-1}\left(1+a_{k}\right)^{-1}: j=1, \ldots, p, l=0, \ldots, p\right\}, \underline{B} \geq \min \left\{\prod_{k=j}^{j+l-1}\left(1+b_{k}\right)^{-1}: j=\right.$ $1, \ldots, p, l=0, \ldots, p\}$.

We stress that there are significant differences between our setting and the situation in [9], where only linear impulses given by functions $I_{k}(u)=b_{k} u$ are allowed: in contrast with [9], where $J_{k}(u) \equiv\left(1+b_{k}\right)^{-1}$ is constant, in the present setting $J_{k}(u)$ depends on $u$, need not be defined at $u=0(k \in \mathbb{N})$ and the functions $B(t ; y)$ and $\tilde{B}(s, t ; y)$ now depend on $y \in X^{+} \backslash\{0\}$.

Consider the partial order in $X$ induced by the cone $X^{+}$, i.e., for $x, y \in X, x \leq y$ means that $y-x \in X^{+}$. For any $\sigma \in(0,1)$, we consider a new cone in $X$, as defined by Nieto in [22] and in many other papers:

$$
K_{\sigma}:=\left\{y \in X^{+}: y(t) \geq \sigma\|y\|\right\} .
$$

For a fixed constant $\sigma \in(0,1)$, we shall refer to $K_{\sigma}$ simply as $K$. Next, we define the operator $\Phi$ on $K \backslash\{0\}$ by

$$
(\Phi y)(t)=\left(B(\omega ; y) \mathrm{e}^{A(\omega)}-1\right)^{-1} \int_{t}^{t+\omega} \tilde{B}(s, t ; y) g\left(s, y_{s}\right) \mathrm{e}^{\int_{t}^{s} a(u) d u} d s, \quad t \geq 0
$$


for $y \in K, y \neq 0$, where, according to the above notation,

$$
A(\omega)=\int_{0}^{\omega} a(t) d t, \quad B(\omega ; y)=\prod_{k=1}^{p} J_{k}\left(y\left(t_{k}\right)\right) .
$$

Remark 2.1. It is worth mentioning that, at each impulse instant $t_{k}$, a solution $y$ of (1.1) satisfies $J_{k}\left(y\left(t_{k}\right)\right)=y\left(t_{k}\right) / y\left(t_{k}^{+}\right)$, i.e., $J_{k}\left(y\left(t_{k}\right)\right)$ gives the ratio between the two lateral limits of $y(t)$ at $t_{k}$. As a consequence, the function $x(t):=B(t ; y) y(t)$ is continuous (as already observed in [29]), and this fact plays an important role, as the fixed points of the operator $\Phi$ turn out to be periodic solutions of (1.1), cf. Lemma 2.3 below.

The construction in the remainder of this section, as well as the proofs of the next lemmas, follow along the main ideas in [9, Section 2], which however have to be carefully adapted, in order to tackle the problems caused by the dependence of the functions $B(t ; y)$ and $\tilde{B}(s, t ; y)$ on $y \in K \backslash\{0\}$, and by the fact that $\Phi$ may not be defined at $y=0$. Most of the arguments are included here, nevertheless the reader can check [9] for some omitted details.

Lemma 2.2. Assume (A1)-(A4) and take $\sigma \leq(\underline{B} / \bar{B}) \mathrm{e}^{-A(\omega)}$. Then $\Phi(K \backslash\{0\}) \subset K$.

Proof. Let $y \in K, y \neq 0$ be fixed. As seen above, $y_{t} \in \tilde{X} \subset P C_{f}$ for $t \geq 0$. The definition of $\Phi$, (A1)-(A4) and a simple change of variables, show that $\Phi y \geq 0$ and that $\Phi$ is $\omega$-periodic. Moreover, it is clear that $t \mapsto(\Phi y)(t)$ is continuous for every $0 \leq t \neq t_{k}$ and left-continuous on $t_{k}, k=1, \ldots, p$.

Take $\varepsilon>0$ with $\varepsilon<\min _{1 \leq k \leq p}\left(t_{k+1}-t_{k}\right), 0 \leq t \leq \omega$ and $k \in\{1, \ldots, p\}$. We have

$$
J_{k}\left(y\left(t_{k}\right)\right) \tilde{B}\left(s, t_{k}+\varepsilon ; y\right)=\tilde{B}\left(s, t_{k} ; y\right),
$$

for any $t_{k}+\varepsilon \leq s \leq t_{k}+\omega$, while $\tilde{B}\left(s, t_{k}+\varepsilon ; y\right)=\tilde{B}(\omega ; y)$ if $t_{k}+\omega<s \leq t_{k}+\omega+\varepsilon$. Hence,

$$
\begin{aligned}
(\Phi y)\left(t_{k}+\varepsilon\right) & =\left(B(\omega ; y) \mathrm{e}^{A(\omega)}-1\right)^{-1} \mathrm{e}^{-\int_{t_{k}}^{t_{k}+\varepsilon} a(u) d u}\left[J_{k}\left(y\left(t_{k}\right)\right)^{-1} \int_{t_{k}+\varepsilon}^{t_{k}+\omega} \tilde{B}\left(s, t_{k} ; y\right) g\left(s, y_{s}\right) \mathrm{e}^{\int_{t_{k}}^{s} a(u) d u} d s\right. \\
& \left.+\tilde{B}(\omega ; y) \int_{t_{k}+\omega}^{t_{k}+\omega+\varepsilon} g\left(s, y_{s}\right) \mathrm{e}^{\int_{t_{k}}^{s} a(u) d u} d s\right] .
\end{aligned}
$$

Since $\tilde{B}(\omega ; y) \leq \bar{B}$ and $g$ is bounded on bounded sets of $\mathbb{R}^{+} \times \tilde{X}$, it follows

$$
0 \leq \tilde{B}(\omega ; y) \int_{t_{k}+\omega}^{t_{k}+\omega+\varepsilon} g\left(s, y_{s}\right) \mathrm{e}^{\int_{t_{k}}^{s} a(u) d u} d s \leq \mathrm{e}^{A(\omega)} \bar{B} \int_{t_{k}+\omega}^{t_{k}+\omega+\varepsilon} g\left(s, y_{s}\right) d s \rightarrow 0 \quad \text { as } \quad \varepsilon \rightarrow 0
$$

By letting $\varepsilon \rightarrow 0^{+}$, we obtain

$$
\Phi y\left(t_{k}^{+}\right)=J_{k}\left(y\left(t_{k}\right)\right)^{-1} \Phi y\left(t_{k}\right) \in \mathbb{R} .
$$

Thus, we conclude that $\Phi(K \backslash\{0\}) \subset X^{+}$.

Now, we check that $\Phi(K \backslash\{0\}) \subset K$. Take $y \in K, y \neq 0$, and $t \geq 0$. Then,

$$
\begin{aligned}
& \|\Phi y\| \leq\left(B(\omega ; y) \mathrm{e}^{A(\omega)}-1\right)^{-1} \bar{B} \mathrm{e}^{A(\omega)} \int_{0}^{\omega} g\left(s, y_{s}\right) d s, \\
& (\Phi y)(t) \geq\left(B(\omega ; y) \mathrm{e}^{A(\omega)}-1\right)^{-1} \underline{B} \int_{0}^{\omega} g\left(s, y_{s}\right) d s .
\end{aligned}
$$


These two inequalities imply that

$$
(\Phi y)(t) \geq(\underline{B} / \bar{B}) \mathrm{e}^{-A(\omega)}\|\Phi y\| \geq \sigma\|\Phi y\|,
$$

which leads to $\Phi(K \backslash\{0\}) \subset K$.

From the former lemma, we can suppose hereafter that $\sigma$ is chosen so that $K=K_{\sigma}$ satisfies $\Phi(K \backslash\{0\}) \subset K$.

Lemma 2.3. Assume (A1)-(A4). Then $y \in K \backslash\{0\}$ is a positive $\omega$-periodic solution of (1.1) if and only if $y$ is a fixed point of $\Phi$.

Proof. Let $y$ be a positive $\omega$-periodic solution to (1.1). As observed in Remark 2.1 the function $x(t):=B(t ; y) y(t)$ is continuous, and it satisfies

$$
x^{\prime}(t)=B(t ; y) y^{\prime}(t)=-a(t) x(t)+B(t ; y) g\left(t, y_{t}\right),
$$

for any $t \geq 0, t \neq t_{k}, k \in\{1, \ldots, p\}$. Integration over $[t, t+\omega]$ leads to

$$
\begin{aligned}
x(t+\omega) e^{A(t+\omega)}-x(t) e^{A(t)} & =\left[B(t+\omega ; y) e^{A(\omega)}-B(t ; y)\right] y(t) e^{A(t)} \\
& =\int_{t}^{t+\omega} B(s ; y) g\left(s, y_{s}\right) e^{A(s)} d s .
\end{aligned}
$$

Since $B(t+\omega ; y)=B(\omega ; y) B(t ; y)$ and $\tilde{B}(s, t ; y)=B(s ; y) B(t ; y)^{-1}$, one obtains

$$
\left(B(\omega ; y) e^{A(\omega)}-1\right) y(t)=\int_{t}^{t+\omega} \tilde{B}(s, t ; y) g\left(s, y_{s}\right) e^{\int_{t}^{s} a(u) d u} d s
$$

and thus $y=\Phi y$. The converse follows along similar lines.

In view of Lemma 2.3, finding positive periodic solutions of (1.1) in a cone $K$ is equivalent to finding fixed points of the operator $\Phi$. To provide criteria for the existence of a fixed point of $\Phi$ in $K \backslash\{0\}$, the Krasnoselskii-Guo fixed point theorem in the version given below will be used.

Theorem 2.1. [11] Let $X$ be a Banach space, $K$ a cone in $X$ and $A_{r, R}:=\{y \in K: r \leq\|y\| \leq R\}$, for some $0<r<R$. Let $T: A_{r, R} \rightarrow K$ be a completely continuous operator. Suppose that there exist $r, R$ with $0<r<R$ such that one of the following forms is satisfied:

(a) compressive form: $\|T y\| \leq R$ if $y \in K,\|y\|=R$ and $\|T y\| \geq r$ if $y \in K,\|y\|=r$;

(b) expansive form: $\|T y\| \leq r$ if $y \in K,\|y\|=r$ and $\|T y\| \geq R$ if $y \in K,\|y\|=R$. Then there exists a fixed point $y^{*}$ of $T$ in $A_{r, R}$.

In order to apply this theorem to our setting, we need to check that $\Phi$ is completely continuous on a conical shell $A_{r, R}$. The proof of the continuity of $\Phi$ requires an extra technical hypothesis:

(A5) The family of operators $g(t, \cdot)$, with $t \in[0, \omega]$, is uniformly equicontinuous on bounded sets of $\tilde{X} \backslash\{0\}$, in the sense that, for any $A \subset \tilde{X} \backslash\{0\}$ bounded and $\varepsilon>0$, there is $\delta=\delta(\varepsilon)>0$ such that $\left|g\left(t, \varphi_{1}\right)-g\left(t, \varphi_{2}\right)\right|<\varepsilon$, for all $t \in[0, \omega]$ and $\varphi_{1}, \varphi_{2} \in A$ with $\left\|\varphi_{1}-\varphi_{2}\right\|<\delta$. 
Note that in (A5) one can replace $\tilde{X}$ by $\tilde{K}:=\left\{y_{t}: y \in K, t \geq 0\right\}$. It is clear that (A5) is satisfied if $g$ is uniformly continuous on bounded sets of $[0, \omega] \times \tilde{X}$, however this latter requirement is too strong for our purposes.

Lemma 2.4. If (A1)-(A5) hold, $\Phi$ is completely continuous on any conical sector $A_{r, R}, 0<r<R$.

Proof. First, we prove that $\Phi$ is continuous on $K \backslash\{0\}$. In view of (A2), the functions $u \mapsto J_{k}(u)$ are continuous on $(0, \infty)$, thus $B(\omega ; y)$ and $\tilde{B}(s, t ; y)$ are continuous in $y \in K \backslash\{0\}$. Recall also that $B(\omega ; y), \tilde{B}(s, t ; y)$ are uniformly bounded from below and above by positive constants $\underline{B}, \bar{B}$ on $D \times\left(X^{+} \backslash\{0\}\right)$, where $D=\{(s, t): t \in[0, \omega], s \in[t, t+\omega]\}$, and $g$ is bounded on bounded sets of $[0, \omega] \times \tilde{X}$.

Fix $y_{*} \in K, y_{*} \neq 0$. For any $\varepsilon \in\left(0,\left\|y_{*}\right\|\right), t \in[0, \omega]$ and $y \in K \cap B_{\varepsilon}\left(y_{*}\right)$, write

$$
\begin{aligned}
\left|\Phi y(t)-\Phi y_{*}(t)\right| & \leq\left|\left(B(\omega ; y) \mathrm{e}^{A(\omega)}-1\right)^{-1}-\left(B\left(\omega ; y_{*}\right) \mathrm{e}^{A(\omega)}-1\right)^{-1}\right| \bar{B} \mathrm{e}^{A(\omega)} \int_{0}^{\omega} g\left(s, y_{s}\right) d s \\
& +\left(B\left(\omega ; y_{*}\right) \mathrm{e}^{A(\omega)}-1\right)^{-1} \mathrm{e}^{A(\omega)} \bar{B} \int_{0}^{\omega}\left|g\left(s, y_{s}\right)-g\left(s, y_{*, s}\right)\right| d s \\
& +\left(B\left(\omega ; y_{*}\right) \mathrm{e}^{A(\omega)}-1\right)^{-1} \mathrm{e}^{A(\omega)} \int_{t}^{t+\omega}\left|\tilde{B}(s, t ; y)-\tilde{B}\left(s, t ; y_{*}\right)\right| g\left(s, y_{*, s}\right) d s .
\end{aligned}
$$

Clearly,

$$
\left(B(\omega ; y) \mathrm{e}^{A(\omega)}-1\right)^{-1}-\left(B\left(\omega ; y_{*}\right) \mathrm{e}^{A(\omega)}-1\right)^{-1} \rightarrow 0 \quad \text { as } \quad y \rightarrow y_{*} .
$$

By induction on $p$, it is easy to prove that

$$
\left|\tilde{B}(s, t ; y)-\tilde{B}\left(s, t ; y_{*}\right)\right| \leq \bar{B} \sum_{k=1}^{p}\left|J_{k}\left(y\left(t_{k}\right)\right)-J_{k}\left(y_{*}\left(t_{k}\right)\right)\right|
$$

for $0 \leq t \leq s \leq t+\omega$. Consequently, there exists $\delta_{1} \in(0, \varepsilon)$ such that, if $\left\|y-y_{*}\right\|<\delta_{1}$, then

$$
\left|\tilde{B}(s, t ; y)-\tilde{B}\left(s, t ; y_{*}\right)\right| \leq \varepsilon \quad \text { for all } \quad(s, t) \in D
$$

and we derive that

$$
\max _{t \in[0, \omega]} \int_{t}^{t+\omega}\left|\tilde{B}(s, t ; y)-\tilde{B}\left(s, t ; y_{*}\right)\right| g\left(s, y_{*, s}\right) d s \rightarrow 0 \quad \text { as } \quad y \rightarrow y_{*} .
$$

Finally, for $R=\varepsilon+\left\|y_{*}\right\|$, from (A5) there exists $\delta_{2} \in(0, \varepsilon)$ such that $\left|g\left(s, \varphi_{1}\right)-g\left(s, \varphi_{2}\right)\right|<\varepsilon \omega^{-1}$ for $s \in[0, \omega]$ and $\varphi_{1}, \varphi_{2} \in \tilde{X} \backslash\{0\}$ with $\left\|\varphi_{1}\right\|,\left\|\varphi_{2}\right\| \leq R$ and $\left\|\varphi_{1}-\varphi_{2}\right\|<\delta_{2}$.

Note also that $\left\|y_{s}-y_{*, s}\right\|=\left\|y-y_{*}\right\|$ for all $s \geq 0$, implying that, for $y \in K \cap B_{\delta_{2}}\left(y_{*}\right)$, we have

$$
\int_{0}^{\omega}\left|g\left(s, y_{s}\right)-g\left(s, y_{*, s}\right)\right| d s<\varepsilon \text {. }
$$

From (2.5) and the above computations, we conclude that $\left\|\Phi y-\Phi y_{*}\right\| \rightarrow 0$ as $\left\|y-y_{*}\right\| \rightarrow 0$.

Next, fix $A_{r, R}$ with $0<r<R$. To show that $\Phi: A_{r, R} \rightarrow K$ is a compact operator, we define the operator

$$
(\mathcal{F} y)(t)=B(t ; y)(\Phi y)(t), \quad y \in K \backslash\{0\}
$$


From formula $(2.4),(\mathcal{F} y)(t)$ is continuous on $[0, \infty)$. Reasoning as in [9, Lemma 2.3], one proves that $\mathcal{F}_{0}:=\left\{\left.(\mathcal{F} y)\right|_{[0, \omega]}: y \in A_{r, R}\right\} \subset C([0, \omega] ; \mathbb{R})$ is bounded and equicontinuous, and this procedure allows us to conclude that $\Phi\left(A_{r, R}\right)$ is relatively compact in $K$. Details are omitted.

Remark 2.2. For the case of DDEs with finite delay (1.3), in [9] a technical condition was also imposed, in order to prove that $\Phi$ is a continuous operator: hypothesis (h5) in [9] requires that the function $(t, y) \mapsto g\left(t, y_{t}\right)$ is uniformly continuous on bounded sets of $[0, \omega] \times K$. However, this condition is not fulfilled by most of the functions $g$, since the map $t \mapsto y_{t}$ is not continuous in the impulsive case; in fact, the additional assumption (h5) in [9] should be replaced by the above requirement (A5).

\section{Existence of positive periodic solutions}

We are now ready to state the main result of the paper. Beforehand, we introduce some further notation, which allows us to simplify the exposition.

Since $J_{k}(u)$ are bounded below and above by positive constants, there exist in $(0, \infty)$

$$
\begin{aligned}
& J_{k}(0)^{s}:=\limsup _{u \rightarrow 0^{+}} J_{k}(u), J_{k}(0)^{i}:=\liminf _{u \rightarrow 0^{+}} J_{k}(u), \\
& J_{k}(\infty)^{s}:=\limsup _{u \rightarrow \infty} J_{k}(u), J_{k}(\infty)^{i}:=\liminf _{u \rightarrow \infty} J_{k}(u), \quad k=1, \ldots, p .
\end{aligned}
$$

We further denote

$$
\begin{aligned}
B^{0} & :=\prod_{k=1}^{p} J_{k}(0)^{s}, B_{0}:=\prod_{k=1}^{p} J_{k}(0)^{i}, \\
B^{\infty}: & =\prod_{k=1}^{p} J_{k}(\infty)^{s}, \quad B_{\infty}:=\prod_{k=1}^{p} J_{k}(\infty)^{i} .
\end{aligned}
$$

For a continuous function $h:[0, \infty) \rightarrow[0, \infty)$, let $L_{i}(h), L^{i}(h) \in[0, \infty], i=0, \infty$, be defined by

$$
\begin{array}{ll}
L_{0}(h)=\liminf _{u \rightarrow 0^{+}} \frac{h(u)}{u}, & L_{\infty}(h)=\liminf _{u \rightarrow \infty} \frac{h(u)}{u} \\
L^{0}(h)=\limsup _{u \rightarrow 0^{+}} \frac{h(u)}{u}, & L^{\infty}(h)=\limsup _{u \rightarrow \infty} \frac{h(u)}{u} .
\end{array}
$$

If it is clear which function $h$ we are referring to, we may simply write $L_{i}, L^{i}$ for $L_{i}(h), L^{i}(h), i=0, \infty$.

In the sequel, we shall impose one of the following assumptions:

(A6) There are constants $r_{0}, R_{0}$ with $0<r_{0}<R_{0}$ and continuous functions $b, h:[0, \infty) \rightarrow[0, \infty)$, with $b(t) \not \equiv 0$ and $\omega$-periodic, such that for $r>0, y \in K$ and $t \geq 0$ it holds:

$$
\begin{aligned}
& g\left(t, y_{t}\right) \leq b(t) h(r) \quad \text { if } R_{0} \leq y \leq r \\
& g\left(t, y_{t}\right) \geq b(t) h(r) \quad \text { if } r \leq y \leq r_{0}
\end{aligned}
$$


(A7) There are constants $r_{0}, R_{0}$ with $0<r_{0}<R_{0}$ and continuous functions $b, h:[0, \infty) \rightarrow[0, \infty)$, with $b(t) \not \equiv 0$ and $\omega$-periodic, such that for $r>0, y \in K$ and $t \geq 0$ it holds:

$$
\begin{array}{ll}
g\left(t, y_{t}\right) \geq b(t) h(r) & \text { if } y \geq r \geq R_{0}, \\
g\left(t, y_{t}\right) \leq b(t) h(r) & \text { if } 0<y \leq r \leq r_{0} .
\end{array}
$$

Theorem 3.1. For (1.1), assume (A1)-(A5). In addition, assume one of the following conditions:

(i) (sublinear case) (A6) holds with b, h satisfying

$$
C_{1} L_{0}>1 \text { and } C_{2} L^{\infty}<1
$$

where $L_{0}=L_{0}(h), L^{\infty}=L^{\infty}(h)$ are as in (3.3) and $C_{i}=C_{i}(b)(i=1,2)$ are given by

$$
\begin{aligned}
& C_{1}(b)=\left(\mathrm{e}^{A(\omega)} B^{0}-1\right)^{-1} \min _{t \in[0, \omega]} \int_{t}^{t+\omega} b(s) \mathrm{e}^{\int_{t}^{s} a(u) d u} \prod_{k: t_{k} \in[t, s)} J_{k}(0)^{i} d s \\
& C_{2}(b)=\left(\mathrm{e}^{A(\omega)} B_{\infty}-1\right)^{-1} \max _{t \in[0, \omega]} \int_{t}^{t+\omega} b(s) \mathrm{e}^{\int_{t}^{s} a(u) d u} \prod_{k: t_{k} \in[t, s)} J_{k}(\infty)^{s} d s
\end{aligned}
$$

(ii) (superlinear case) (A7) holds with b, h satisfying

$$
C_{4} L^{0}<1 \text { and } C_{3} L_{\infty}>1
$$

where $L^{0}=L^{0}(h), L_{\infty}=L_{\infty}(h)$ are as in (3.3) and $C_{i}=C_{i}(b)(i=3,4)$ are given by

$$
\begin{aligned}
& C_{3}(b)=\left(\mathrm{e}^{A(\omega)} B^{\infty}-1\right)^{-1} \min _{t \in[0, \omega]} \int_{t}^{t+\omega} b(s) \mathrm{e}^{\int_{t}^{s} a(u) d u} \prod_{k: t_{k} \in[t, s)} J_{k}(\infty)^{i} d s, \\
& C_{4}(b)=\left(\mathrm{e}^{A(\omega)} B_{0}-1\right)^{-1} \max _{t \in[0, \omega]} \int_{t}^{t+\omega} b(s) \mathrm{e}^{\int_{t}^{s} a(u) d u} \prod_{k: t_{k} \in[t, s)} J_{k}(0)^{s} d s .
\end{aligned}
$$

Then there exists at least one positive $\omega$-periodic solution of (1.1).

Proof. We shall use Theorem 2.1, to conclude that $\Phi: A_{r, R} \rightarrow K$ has a fixed point in a conical sector $A_{r, R}:=\{y \in K: r \leq\|y\| \leq R\}$, for some $0<r<R$.

First, suppose the sublinear case (i) is satisfied. From (A4), (2.2), (3.1) and $C_{2} L^{\infty}<1$, where $C_{2}$ is as in (3.7), one can choose $\varepsilon>0$ sufficiently small so that $\mathrm{e}^{A(\omega)}\left(B_{\infty}-\varepsilon\right)-1>0$ and $C_{2}(\varepsilon) L^{\infty}<1$, where

$$
C_{2}(\varepsilon)=\left(\mathrm{e}^{A(\omega)}\left(B_{\infty}-\varepsilon\right)-1\right)^{-1} \max _{t \in[0, \omega]} \int_{t}^{t+\omega}\left(\prod_{k: t_{k} \in[t, s)} J_{k}(\infty)^{s}+\varepsilon\right) b(s) \mathrm{e}^{\int_{t}^{s} a(u) d u} d s .
$$

Take $R_{1} \geq R_{0}$ such that

$$
\prod_{k=j}^{j+l-1} J_{k}\left(u_{k}\right) \leq \prod_{k=j}^{j+l-1} J_{k}(\infty)^{s}+\varepsilon \quad \text { for } u_{k} \geq R_{1}, k \in\{j, \ldots, j+l-1\}, j, l=1, \ldots, p,
$$


and

$$
\prod_{k=1}^{p} J_{k}\left(u_{k}\right) \geq B_{\infty}-\varepsilon \quad \text { for } \quad u_{1}, \ldots, u_{p} \geq R_{1} .
$$

For any $R \geq \sigma^{-1} R_{1}$ and $y \in K$ such that $\|y\|=R$, from the definition of $K$ we obtain that $R_{0} \leq R_{1} \leq y(t) \leq R, \tilde{B}(s, t ; y) \leq \prod_{k: t_{k} \in[t, s)} J_{k}(\infty)^{s}+\varepsilon$, thus the first inequality in (3.4) implies

$$
\begin{aligned}
(\Phi y)(t) & \leq\left(\mathrm{e}^{A(\omega)}\left(B_{\infty}-\varepsilon\right)-1\right)^{-1} h(R) \int_{t}^{t+\omega}\left(\prod_{k: t_{k} \in[t, s)} J_{k}(\infty)^{s}+\varepsilon\right) b(s) \mathrm{e}^{\int_{t}^{s} a(u) d u} d s \\
& \leq h(R) C_{2}(\varepsilon),
\end{aligned}
$$

for $t \in[0, \omega]$. Since $C_{2}(\varepsilon) L^{\infty}<1$, there exists $M \in \mathbb{R}$ such that $C_{2}(\varepsilon) \frac{h(u)}{u}<1$ for $u \geq M$, hence we can choose any $R \geq \max \left\{M, \sigma^{-1} R_{1}\right\}$, to obtain

$$
\|\Phi y\|<R \quad \text { for } \quad y \in K,\|y\|=R .
$$

Now, from $C_{1} L_{0}>1$, where $C_{1}$ is as in (3.7), one can choose $\varepsilon>0$ such that $\prod_{k=j}^{j+l-1} J_{k}(0)^{i}-\varepsilon>$ $0(1 \leq j, l \leq p)$ and $C_{1}(\varepsilon) L_{0}>1$, where

$$
C_{1}(\varepsilon)=\left(\mathrm{e}^{A(\omega)}\left(B^{0}+\varepsilon\right)-1\right)^{-1} \min _{t \in[0, \omega]} \int_{t}^{t+\omega}\left(\prod_{k: t_{k} \in[t, s)} J_{k}(0)^{i}-\varepsilon\right) b(s) \mathrm{e}^{\int_{t}^{s} a(u) d u} d s .
$$

If one considers $r \in\left(0, r_{0}\right)$ small enough so that $C_{1}(\varepsilon) \frac{h(u)}{u}>1$, if $0<u \leq r$,

$$
\prod_{k=j}^{j+l-1} J_{k}\left(u_{k}\right) \geq \prod_{k=j}^{j+l-1} J_{k}(0)^{i}-\varepsilon \quad \text { for } \quad u_{k} \leq r, k \in\{j, \ldots, j+l-1\}, j, l=1, \ldots, p
$$

and

$$
\prod_{k=1}^{p} J_{k}\left(u_{k}\right) \leq B^{0}+\varepsilon \quad \text { for } 0<u_{1}, \ldots, u_{p} \leq r
$$

from the second condition in (3.4), for $y \in K$ with $0<\|y\| \leq r$, it holds

$$
\begin{aligned}
(\Phi y)(t) & \geq\left(\mathrm{e}^{A(\omega)}\left(B^{0}+\varepsilon\right)-1\right)^{-1} h(r) \int_{t}^{t+\omega}\left(\prod_{k: t_{k} \in[t, s)} J_{k}(0)^{i}-\varepsilon\right) b(s) \mathrm{e}^{\int_{t}^{s} a(u) d u} d s \\
& \geq h(r) C_{1}(\varepsilon)>r, \quad \text { for } t \in[0, \omega] .
\end{aligned}
$$

Thus, $\|\Phi y\|>r$. From Theorem 2.1(a), we conclude that there exists at least one $\omega$-periodic solution of (1.1) in the sector $A_{r, R}$.

For the superlinear case (ii), the proof follows from Theorem 2.1(b) by arguing in a similar way, as the reader can easily check. To avoid repetitions, we do not include it here.

Remark 3.1. As in e.g. [1, 10, 19, 21, 35], under suitable hypotheses, a combination of both the compressive and expansive forms of Krasnoselskii cone theorem (see [11, 16]) can lead to the existence of more than one positive period solution to (1.1). 
Remark 3.2. It is apparent that the above method can be extended to other families of impulsive scalar DDEs. For instance, under the same general assumptions (A1)-(A3),(A5), and with (A4) replaced by $\prod_{i=1}^{p}\left(1+a_{k}\right)>\exp \left(-\int_{0}^{\omega} a(t) d t\right)$, Theorem 3.1 applies to impulsive DDEs where the equation $x^{\prime}(t)=-a(t) x(t)+g\left(t, x_{t}\right)$ is replaced by $x^{\prime}(t)=a(t) x(t)-g\left(t, x_{t}\right)$. By straightforward adjustments, the above technique can also be used to treat DDEs in the more general form $x^{\prime}(t)=$ $(-1)^{i}\left[-a(t) x(t) g_{0}(t, x(t))+g\left(t, x_{t}\right)\right]$, with $i=1,2, g_{0}$ continuous, nonnegative and bounded above and below by positive constants, and subject to impulses as in (1.1). For results along these lines, see $[2,21,30,34]$. Extensions to DDEs where $g(t, \varphi)$ has a singularity at $\varphi=0$ are also feasible, since the operator $\Phi$ in (2.3) is not required to be defined at $y=0$.

Whenever either (A6) or (A7) is satisfied by some functions $b, h$, now we would like to establish alternative criteria for the existence of a positive periodic solution based on either a pointwise or an average comparison between $a(t)$ and $b(t)$. Of course, the contribution of the impulses has to be taken into account. Some results using a pointwise comparison between $a(t)$ and $g(t, \cdot)$ can be found in $[2,9,18,19,27]$, where typically a restriction $b(t)>a(t)$ is imposed. The second approach, relating the integral averages of $a(t), b(t)$ over $[0, \omega]$, has rarely been used in the literature, even for the case of DDEs without impulses; see [4, 9, 21]. We explore these ideas separately for the sublinear and the superlinear cases in the next corollaries, which turn out to be very usual in applications.

Corollary 3.1. (sublinear case) Consider (1.1) and suppose that (A1)-(A6) hold. For functions b, $h$ as in (A6), let $L_{0}=L_{0}(h), L^{\infty}=L^{\infty}(h)$ be as in (3.3) and define $\underline{B}(0)=\min \left\{\prod_{k=j}^{j+l-1} J_{k}(0)^{i}: 1 \leq\right.$ $j \leq p, 0 \leq l \leq p\}, \bar{B}(\infty)=\max \left\{\prod_{k=j}^{j+l-1} J_{k}(\infty)^{s}: 1 \leq j \leq p, 0 \leq l \leq p\right\}$. Assume in addition that:

(a) either

$$
m_{1} C_{1}^{*} L_{0}>1, \quad m_{2} C_{2}^{*} L^{\infty}<1,
$$

where $m_{1}, m_{2}>0$ are such that $m_{1} a(t) \leq b(t) \leq m_{2} a(t)$ for $t \in[0, \omega]$ and

$$
C_{1}^{*}=\left(\mathrm{e}^{A(\omega)} B^{0}-1\right)^{-1} \underline{B}(0)\left(\mathrm{e}^{A(\omega)}-1\right), \quad C_{2}^{*}=\left(\mathrm{e}^{A(\omega)} B_{\infty}-1\right)^{-1} \bar{B}(\infty)\left(\mathrm{e}^{A(\omega)}-1\right) ;
$$

(b) or

$$
C_{1}^{* *} L_{0} \geq 1, \quad C_{2}^{* *} \mathrm{e}^{A(\omega)} L^{\infty} \leq 1
$$

where

$$
C_{1}^{* *}=\left(\mathrm{e}^{A(\omega)} B^{0}-1\right)^{-1} \underline{B}(0) \int_{0}^{\omega} b(t) d t, \quad C_{2}^{* *}=\left(\mathrm{e}^{A(\omega)} B_{\infty}-1\right)^{-1} \bar{B}(\infty) \int_{0}^{\omega} b(t) d t .
$$

Then there exists at least one positive $\omega$-periodic solution of (1.1).

Proof. If $m_{1} a(t) \leq b(t) \leq m_{2} a(t)$ for $t \in[0, \omega]$, we have

$$
m_{1} \int_{t}^{t+\omega} a(s) \mathrm{e}^{\int_{t}^{s} a(u) d u} d s \leq \int_{t}^{t+\omega} b(s) \mathrm{e}^{\int_{t}^{s} a(u) d u} d s \leq m_{2} \int_{t}^{t+\omega} a(s) \mathrm{e}^{\int_{t}^{s} a(u) d u} d s
$$

and $\int_{t}^{t+\omega} a(s) \mathrm{e}^{\int_{t}^{s} a(u) d u} d s=\mathrm{e}^{A(\omega)}-1$. For $C_{i}$ and $C_{i}^{*}, i=1,2$, given by (3.7) and (3.13), we get the estimates

$$
C_{1} \geq m_{1} C_{1}^{*} \quad \text { and } \quad C_{2} \leq m_{2} C_{2}^{*}
$$


and the result follows from Theorem 3.1(a).

On the other hand, since $a(t) \not \equiv 0, \int_{0}^{\omega} b(s) d s<\int_{t}^{t+\omega} b(s) \mathrm{e}^{\int_{t}^{s} a(u) d u} d s<\mathrm{e}^{A(\omega)} \int_{0}^{\omega} b(s) d s$ for $t \in[0, \omega]$, thus from (b) and the definition of $C_{i}, C_{i}^{* *}, i=1,2$, it is clear that

$$
C_{1}>C_{1}^{* *} \text { and } C_{2}<C_{2}^{* *} \mathrm{e}^{A(\omega)} .
$$

Again from Theorem 3.1(a), we obtain the result.

Remark 3.3. In (a) of the above corollary, if $b(t)-m_{1} a(t) \geq 0$, respectively $m_{2} a(t)-b(t) \geq 0$, is not identically zero, in (3.12) one can replace the strict inequality $m_{1} C_{1}^{*} L_{0}>1$ by $m_{1} C_{1}^{*} L_{0} \geq 1$, respectively $m_{2} C_{2}^{*} L^{\infty}<1$ by $m_{2} C_{2}^{*} L^{\infty} \leq 1$. Also, if (3.4) is fulfilled with $b(t)=a(t)$, condition (3.12) reduces to $C_{2}^{*} L^{\infty}<1<C_{1}^{*} L_{0}$. Moreover, obviously in (3.13), (3.15) one can replace $\underline{B}(0)$ by $\underline{B}$ and $\bar{B}(\infty)$ by $\bar{B}$.

Similar arguments and Theorem 3.1(b) lead to:

Corollary 3.2. (superlinear case) Consider (1.1) and suppose that (A1)-(A5) and (A7) hold. For functions $b, h$ as in (A7), let $L^{0}=L^{0}(h), L_{\infty}=L_{\infty}(h)$ be as in $(3.3), \bar{B}(0)=\max \left\{\prod_{k=j}^{j+l-1} J_{k}(0)^{u}\right.$ : $1 \leq j \leq p, 0 \leq l \leq p\}, \underline{B}(\infty)=\min \left\{\prod_{k=j}^{j+l-1} J_{k}(\infty)^{i}: 1 \leq j \leq p, 0 \leq l \leq p\right\}$ and further define $C_{i}^{*}$ and $C_{i}^{* *}=C_{i}^{* *}(b)(i=3,4)$ by

$$
\begin{aligned}
& C_{3}^{*}=\left(\mathrm{e}^{A(\omega)} B^{\infty}-1\right)^{-1} \underline{B}(\infty)\left(\mathrm{e}^{A(\omega)}-1\right), \quad C_{4}^{*}=\left(\mathrm{e}^{A(\omega)} B_{0}-1\right)^{-1} \bar{B}(0)\left(\mathrm{e}^{A(\omega)}-1\right), \\
& C_{3}^{* *}=\left(\mathrm{e}^{A(\omega)} B^{\infty}-1\right)^{-1} \underline{B}(\infty) \int_{0}^{\omega} b(t) d t, \quad C_{4}^{* *}=\left(\mathrm{e}^{A(\omega)} B_{0}-1\right)^{-1} \bar{B}(0) \int_{0}^{\omega} b(t) d t .
\end{aligned}
$$

Then (1.1) has at least one positive $\omega$-periodic solution if one of the following conditions is satisfied:

(a) $m_{2} C_{4}^{*} L^{0}<1<m_{1} C_{3}^{*} L_{\infty}$, where $m_{1}, m_{2}$ are such that $m_{1} a(t) \leq b(t) \leq m_{2} a(t)$ for $t \in[0, \omega]$;

(b) $C_{4}^{* *} \mathrm{e}^{A(\omega)} L^{0} \leq 1 \leq C_{3}^{* *} L_{\infty}$.

Remark 3.4. Note that the constants in (3.13) and (3.17) do not depend explicitly on the function $b(t)$, but this dependence appears in the choice of $m_{1}, m_{2}$. In fact, if $a(t)>0$ on $[0, \omega]$, in (a) of Corollaries 3.1 and 3.2 one may take $m_{1}=\min _{t \in[0, \omega]} \frac{b(t)}{a(t)}, m_{2}=\max _{t \in[0, \omega]} \frac{b(t)}{a(t)}$.

Remark 3.5. If $\lim _{u \rightarrow \infty} \frac{h(u)}{u}=0$, then condition (3.6) reduces to $C_{1} L_{0}>1$; by replacing $h(u)$ by $\bar{h}(u)=C_{1} h(u)$ and $b(t)$ by $\bar{b}(t)=C_{1}^{-1} b(t)$, this latter condition reads as $L_{0}=L_{0}(\bar{h})>1$. In an analogous way, if $\lim _{u \rightarrow 0} \frac{h(u)}{u}=\infty$, then (3.6) reduces to $C_{2} L^{\infty}<1$, and, rescaling the functions $b, h$, this latter requirement is given by $L^{\infty}=L^{\infty}(\bar{h})<1$ for $\bar{h}(u)=C_{2} h(u), \bar{b}(t)=C_{2}^{-1} b(t)$. Similar considerations can be given for the superlinear case when either $\lim _{u \rightarrow \infty} \frac{h(u)}{u}=\infty$ or $\lim _{u \rightarrow 0} \frac{h(u)}{u}=0$. Hence, when one (or more) of the limits $L_{i}$ or $L^{i}$ is 0 or $\infty$, the above criteria can be simplified.

From the viewpoint of applications, the sublinear case is more useful. Note also that for many models from biomathematics the nonlinearity $g$ has a strictly "sublinear" growth at $\infty$, thus $L^{\infty}=0$ (e.g. this always happens if $g$ is bounded). We portray this situation below.

Corollary 3.3. Consider (1.1), assume that (A1)-(A6) hold, with functions $b, h$ in (A6) such that $\lim _{u \rightarrow \infty} \frac{h(u)}{u}=0$ and, for $C_{1}^{*}, C_{1}^{* *}$ as in (3.13), (3.15), one of the following conditions is satisfied:

(a) $b(t) \geq a(t), t \in[0, \omega]$, and $C_{1}^{*} L_{0}>1$; (b) $C_{1}^{* *} L_{0} \geq 1$.

Then, (1.1) has a positive $\omega$-periodic solution. 
Next, we use the framework above to derive results for the non-impulsive version of (1.1):

$$
x^{\prime}(t)=-a(t) x(t)+g\left(t, x_{t}\right), \quad t \geq 0,
$$

which is the particular case of (1.1) with $I_{k} \equiv 0$, for $k \in \mathbb{N}$. In this situation, $X$ is simply the space of continuous and $\omega$-periodic functions $y: \mathbb{R} \rightarrow \mathbb{R}$, endowed with the supremum norm. For (3.19), Theorem 3.1 reads as follows:

Theorem 3.2. Consider (3.19), assume (A1), (A5) and one of the following sets of requirements:

(a) (A6) holds with $b, h$ satisfying $C_{\min } L_{0}>1, C_{\max } L^{\infty}<1$;

(b) (A7) holds with b, h satisfying $C_{\max } L^{0}<1, C_{\min } L_{\infty}>1$; here, $L_{i}=L_{i}(h), L^{i}=L^{i}(h)(i=0, \infty)$ and $C_{\min }=C_{\min }(b), C_{\max }=C_{\max }(b)$ are defined by

$$
\begin{aligned}
& C_{\min }=\left(\mathrm{e}^{A(\omega)}-1\right)^{-1} \min _{t \in[0, \omega]} \int_{t}^{t+\omega} b(s) \mathrm{e}^{\int_{t}^{s} a(u) d u} d s, \\
& C_{\max }=\left(\mathrm{e}^{A(\omega)}-1\right)^{-1} \max _{t \in[0, \omega]} \int_{t}^{t+\omega} b(s) \mathrm{e}^{\int_{t}^{s} a(u) d u} d s .
\end{aligned}
$$

Then (3.19) has at least one positive $\omega$-periodic solution.

The non-impulsive versions of the corollaries above are easily derived. For the sake of illustration, here we only write Corollary 3.1 for the situation without impulses, for which $C_{1}^{*}=C_{2}^{*}=1$ and $C_{1}^{* *}=C_{2}^{* *}=\left(e^{A(\omega)}-1\right)^{-1} \int_{0}^{\omega} b(t) d t$.

Corollary 3.4. Consider (3.19) and assume (A1), (A5), (A6), where b, h in (A6) are such that:

(a) either $m_{1} a(t) \leq b(t) \leq m_{2} a(t)$ for $t \in[0, \omega]$ and $m_{2} L^{\infty}<1<m_{1} L_{0}$;

(b) or $L_{0} \int_{0}^{\omega} b(t) d t \geq \mathrm{e}^{A(\omega)}-1, L^{\infty} \int_{0}^{\omega} b(t) d t \leq 1-\mathrm{e}^{-A(\omega)}$.

Then (3.19) admits at least one positive $\omega$-periodic solution.

We end this section with a few examples, to which our criteria are applied and analysed within the context of related literature.

Example 3.1. In [27], Wan et al. considered the following family of non-impulsive scalar DDEs with a single discrete delay:

$$
x^{\prime}(t)=-a(t) x(t)+f(t, x(t-\tau(t))), t \geq 0,
$$

where $a(t)>0, \tau(t) \geq 0, f(t, u) \geq 0$ are continuous and $\omega$-periodic in $t$. This equation has the form (3.19), for $g(t, \varphi)=f(t, \varphi(-\tau(t)))$. In order to conclude the existence of an $\omega$-periodic solution, in [27, Theorem 2.1] the authors prescribed the following sufficient conditions on $f$ :

$$
f_{0}:=\liminf _{u \rightarrow 0^{+}} \min _{t \in[0, \omega]} \frac{f(t, u)}{a(t) u}>1, \quad f^{\infty}:=\limsup _{u \rightarrow \infty} \max _{t \in[0, \omega]} \frac{f(t, u)}{a(t) u}<1 .
$$

Note that, with (3.22) satisfied, there exist constants $\varepsilon, M, \alpha, \beta$ with $M>\varepsilon>0$ and $f^{\infty} \leq \beta<$ $1, f_{0} \geq \alpha>1$ such that $f(t, u) \geq a(t) \alpha u, 0<u<\varepsilon$ and $f(t, u) \leq a(t) \beta u, u>M$. Consequently, Corollary 3.4(a) applies with $b(t)=a(t), m_{1}=m_{2}=1$ and $h: \mathbb{R}^{+} \rightarrow \mathbb{R}^{+}$continuous and such that

$$
h(u)= \begin{cases}\alpha u, & 0<u<\varepsilon \\ \beta u, & u>M\end{cases}
$$


With our notations, $L_{0}=\alpha>1>L^{\infty}=\beta$, thus the result in [27] is generalised here via Corollary 3.4(a).

On the other hand, Amster and Idels [2] considered (3.21) with a possible state dependent delay $\tau(t)=\sigma(t, x(t))$. It was shown in [2, Theorem 2.6] that (3.21) has a positive $\omega$-periodic solution if

$$
\gamma_{\infty}:=\limsup _{u \rightarrow \infty} \max _{t \in[0, \omega]} \frac{f(t, u)}{u}<a(t)<\gamma_{0}:=\liminf _{u \rightarrow 0^{+}} \min _{t \in[0, \omega]} \frac{f(t, u)}{u} \text { for } t \in[0, \omega] .
$$

For $f_{0}, f^{\infty}$ as in (3.22), we have $f_{0} \geq \gamma_{0} / a(t)$ and $f^{\infty} \leq \gamma_{\infty} / a(t)$ for $t \in[0, \omega]$. Clearly, $\gamma_{\infty}<a(t)<\gamma_{0}$ implies $f^{\infty}<1<f_{0}$, thus this criterion is again a particular case of Corollary 3.4(a).

Example 3.2. In [19], the impulsive version of (3.21) was considered (see also [30, 34]):

$$
\begin{cases}x^{\prime}(t)=-a(t) x(t)+f(t, x(t-\tau(t))), & t \geq 0, t \neq t_{k}, k \in \mathbb{N} \\ x\left(t_{k}^{+}\right)-x\left(t_{k}\right)=I_{k}\left(x\left(t_{k}\right)\right), & k \in \mathbb{N}\end{cases}
$$

where $a(t), f(t, u), \tau(t)$ are as in (3.21) and all the impulses are given by nonnegative continuous functions $I_{k}(u)$ satisfying (A2). For $b(t)=a(t)$ and $h(u)$ as in (3.23), from the arguments above and Corollary 3.1(a) we get the existence of at least one positive periodic solution if $f^{\infty} C_{2}^{*}<1<f_{0} C_{1}^{*}$.

As verified in many applications, next suppose also that $f^{\infty}=0$.

Proposition 3.1. For the impulsive DDE (3.24), with $a(t)>0, \tau(t) \geq 0, f(t, u) \geq 0, I_{k}(u) \geq 0$ continuous and $\omega$-periodic in $t$, for $t, u \in \mathbb{R}^{+}$, assume (A2)-(A4) and $f^{\infty}=0$. If

$$
f_{0}\left(\mathrm{e}^{A(\omega)}-1\right) B_{0}>\mathrm{e}^{A(\omega)} B^{0}-1,
$$

then there exists at least one positive $\omega$-periodic solution. In particular, this holds if $f_{0} B_{0}>1$.

Proof. From (3.25), choose $\alpha$ such that $f_{0}>\alpha$ and $\alpha\left(\mathrm{e}^{A(\omega)}-1\right) B_{0}>\mathrm{e}^{A(\omega)} B^{0}-1$, define $b(t)=a(t)$ and $h(u)$ as in (3.23). As observed above, we only need to verify that $\alpha C_{1}^{*}>1$ for $C_{1}^{*}$ as in (3.13). Since $I_{k}(u) \geq 0$ for $u>0$, then $J_{k}(u) \leq 1$ for all $k, \underline{B}(0)=B_{0}$, and therefore

$$
C_{1}^{*}=\left(\mathrm{e}^{A(\omega)}-1\right)\left(\mathrm{e}^{A(\omega)} B^{0}-1\right)^{-1} B_{0},
$$

The above choice of $\alpha$ implies $\alpha C_{1}^{*}>1$. Note also that $B^{0} \leq 1$, thus $C_{1}^{*} \geq B_{0}$.

For the impulsive $\operatorname{DDE}(3.24)$, with $a(t), f(t, u), \tau(t)$ as above and $I_{k}(u)$ nonnegative functions satisfying (A2), Li et al. [19] studied both the sublinear and superlinear cases. For the sublinear situation, Theorem 2.3(i) in [19] asserts that (3.24) has a positive $\omega$-periodic solution provided that

$$
f_{0}+\left(\mathrm{e}^{A(\omega)}-1\right)^{-1} I_{0}>1 \text { and } f^{\infty}+\mathrm{e}^{A(\omega)}\left(\mathrm{e}^{A(\omega)}-1\right)^{-1} I^{\infty}<1
$$

where $f_{0}$ and $f^{\infty}$ are already defined by (3.22) and

$$
I_{0}=\liminf _{u \rightarrow 0^{+}} \sum_{k=1}^{p} \frac{I_{k}(u)}{u}, \quad I^{\infty}=\limsup _{u \rightarrow \infty} \sum_{k=1}^{p} \frac{I_{k}(u)}{u} .
$$


Similar results can be found in [30]. In addition, suppose e.g. $f^{\infty}=0$ and also that $J_{k}(0)^{i}=$ $J_{k}(0)^{s}=\lim _{u \rightarrow 0^{+}} J_{k}(u)$ for $k=1, \ldots, p$. In this setting, we observe that only the first condition in (3.27) is more restrictive than (3.25). In fact, since $I_{k}(u) \geq 0$ and $B_{0}=B^{0}$, we have

$$
1+\sum_{k=1}^{p} \frac{I_{k}(u)}{u} \leq \prod_{k=1}^{p}\left(1+\frac{I_{k}(u)}{u}\right)
$$

which implies $1+I_{0} \leq\left(B^{0}\right)^{-1}$. Thus, for $C_{1}^{*}$ in $(3.26)$ we get

$$
\begin{aligned}
\frac{1}{C_{1}^{*}}-\left[1-\left(\mathrm{e}^{A(\omega)}-1\right)^{-1} I_{0}\right] & =\left(\mathrm{e}^{A(\omega)}-1\right)^{-1}\left[\left(\mathrm{e}^{A(\omega)} B^{0}-1\right) B_{0}^{-1}-\left(\mathrm{e}^{A(\omega)}-1-I_{0}\right)\right] \\
& =\left(\mathrm{e}^{A(\omega)}-1\right)^{-1}\left[-\left(B^{0}\right)^{-1}+1+I_{0}\right] \leq 0,
\end{aligned}
$$

which shows that $f_{0}+\left(\mathrm{e}^{A(\omega)}-1\right)^{-1} I_{0}>1$ implies $f_{0} C_{1}^{*}>1$. In conclusion, the criterion in Proposition 3.1 is proven under less restrictions than in [19].

Remark 3.6. Due to the nature of our operator $\Phi$, where the impulses intervene in a multiplicative mode (rather than additive as in [19,30,34] and many other papers) by means of the products of the auxiliary functions $J_{k}(u)$, the major results given here and in [19] are not always comparable. In [19], a key idea is that e.g. a decay at 0 faster than linear can be tackled with an appropriate choice of positive impulses. For example, if one chooses

$$
g(t, \varphi)=a(t) \varphi(-\tau(t))^{2} \mathrm{e}^{-\varphi(-\tau(t))},
$$

so that $g(t, \varphi)=f(t, \varphi(-\tau(t)))$ for $f(t, u):=a(t) u^{2} \mathrm{e}^{-u}$ and functions $a(t), \tau(t)$ as in (3.24), we have $f_{0}=0, f^{\infty}=0$; from (3.27), in [19] the authors established the existence of $\omega$-periodic orbits for (3.24) provided that

$$
I_{0}>\mathrm{e}^{A(\omega)}-1 \text { and } I^{\infty}<1-\mathrm{e}^{-A(\omega)},
$$

whereas in this situation we are not able to apply Theorem 3.1. On the other hand, as pointed out in Remark 3.1, when $L_{0}=0, L^{\infty}=0$, one could introduce impulses in such a way that Krasnoselskii's results yield the existence of two fixed points of $\Phi$ in a suitable conical sector $A_{r, R} \subset K$. In conclusion, our Theorem 3.1 is not a proper extension of the main results in [19], however our criteria apply to equations which are much more general than (3.24), and with impulses which may change sign.

\section{Applications to delayed Volterra integro-differential equations}

In this section, we apply the results of Section 3 to some Volterra integro-differential equations which are based on well-known models from mathematical biology. In view of the applications, we will focus on consequences of the sublinear case of Theorem 3.1, and on equations with distributed, possibly unbounded delay, since the situation of (multiple) discrete delays was largely illustrated in [9]. We begin with a general setting of Volterra integro-differential equations.

Consider a DDE with distributed delay and nonlinear impulses of the form

$$
\left\{\begin{array}{l}
x^{\prime}(t)=-a(t) x(t)+\int_{-\infty}^{0} k(t, s) F(t, s, x(t+s)) d s, \quad t \geq 0, t \neq t_{k}, k \in \mathbb{N}, \\
x\left(t_{k}^{+}\right)-x\left(t_{k}\right)=I_{k}\left(x\left(t_{k}\right)\right), \quad k \in \mathbb{N},
\end{array}\right.
$$


where:

(h1) $a:[0, \infty) \rightarrow[0, \infty)$ is continuous, $\omega$-periodic and $a \not \equiv 0$;

(h2) $k:[0, \infty) \times(-\infty, 0] \rightarrow[0, \infty)$ is measurable, $\omega$-periodic and continuous in $t$, and $k\left(t_{*}, \cdot\right) \not \equiv 0$ a.e. on $(-\infty, 0]$, for some $t_{*}$; furthermore, for any $t_{0} \in[0, \omega]$ there exist a constant $\varepsilon=\varepsilon\left(t_{0}\right)>0$ and a function $K_{t_{0}}(s)$ Lebesgue integrable on $(-\infty, 0]$, such that

$$
k(t, s) \leq K_{t_{0}}(s) \quad \text { for } \quad t \in I_{\varepsilon}\left(t_{0}\right):=\left[t_{0}-\varepsilon, t_{0}+\varepsilon\right]
$$

(h3) $F:[0, \infty) \times(-\infty, 0] \times[0, \infty) \rightarrow[0, \infty)$ is continuous, $\omega$-periodic in the first variable and $F \not \equiv 0$; furthermore, $F(t, s, x)$ is bounded and uniformly equicontinuous with respect to the variable $x$, on sets $[0, \omega] \times(-\infty, 0] \times A$ for any bounded set $A \subset[0, \infty)$ : i.e., for any $L>0$ and $\varepsilon>0$, there exist $M>0$ and $\delta>0$ such that, for all $(t, s) \in[0, \omega] \times(-\infty, 0]$ and $x_{1}, x_{2} \in[0, L]$ with $\left|x_{1}-x_{2}\right|<\delta$

$$
\left|F\left(t, s, x_{1}\right)\right| \leq M \quad \text { and } \quad\left|F\left(t, s, x_{1}\right)-F\left(t, s, x_{2}\right)\right|<\varepsilon .
$$

For (4.1), we shall also assume that the impulsive assumptions (A2)-(A4) are satisfied.

Under (h2), the functions $s \mapsto k(t, s)$ are summable on $(-\infty, 0]$, and

$$
b(t):=\int_{-\infty}^{0} k(t, s) d s, \quad t \geq 0
$$

is continuous. Of course, $b(t)$ is also nonnegative, $\omega$-periodic and $b\left(t_{*}\right)>0$ for some $t_{*}$. Together with (4.1), we shall also consider its non-impulsive version

$$
x^{\prime}(t)=-a(t) x(t)+\int_{-\infty}^{0} k(t, s) F(t, s, x(t+s)) d s, \quad t \geq 0 .
$$

System (4.1) has the form of our general model (1.1), with the nonlinearity given by

$$
g(t, \varphi)=\int_{-\infty}^{0} k(t, s) F(t, s, \varphi(s)) d s, \quad t \in \mathbb{R}^{+}, \varphi \in \tilde{X}
$$

and is sufficiently general to encompass models with both discrete and distributed delays. Without loss of generality, suppose that $g \not \equiv 0$.

Lemma 4.1. Under (h1)-(h3), a(t) and the nonlinearity $g(t, \varphi)$ in (4.5) satisfy (A1) and (A5).

Proof. Fix $L>0$. From (h3), there is $M>0$ such that $0 \leq F(t, s, \varphi(s)) \leq M$ for $t \in[0, \omega], s \in \mathbb{R}^{-}$ and $\varphi \in \tilde{X}$ with $\|\varphi\| \leq L$, thus $0 \leq g(t, \varphi) \leq M \max _{t \in[0, \omega]} b(t)$, which proves that $g$ is well-defined on $\mathbb{R} \times \tilde{X}$ and bounded on $\mathbb{R} \times\left(\tilde{X} \cap \bar{B}_{L}(0)\right)$.

We now observe that, for any fixed $\varphi \in \tilde{X}$ with $\|\varphi\| \leq L$, the map $t \mapsto g(t, \varphi)$ is continuous on $[0, \omega]$. In fact, for $t, t_{0} \in[0, \omega]$ and $s \in \mathbb{R}^{-}, k(t, s) F(t, s, \varphi(s)) \rightarrow k\left(t_{0}, s\right) F\left(t_{0}, s, \varphi(s)\right)$ as $t \rightarrow t_{0}$, with $0 \leq k(t, s) F(t, s, \varphi(s)) \leq M K_{t_{0}}(s)$ for $t \in I_{\varepsilon}\left(t_{0}\right)$, where $K_{t_{0}}$ is as in (h2). By the Lesbesgue dominated convergence theorem, we have $g(t, \varphi) \rightarrow g\left(t_{0}, \varphi\right)$ as $t \rightarrow t_{0}$. 
Next, fix $\varepsilon>0$. Since the family $\left\{F(t, s, \cdot):(t, s) \in[0, \omega] \times \mathbb{R}^{-}\right\}$is uniformly equicontinuous on $[0, L]$, there is $\delta>0$ such that, for any $t \in[0, \omega], s \in \mathbb{R}^{-}$and $\varphi_{1}, \varphi_{2} \in \tilde{X} \cap \bar{B}_{L}(0)$ with $\left\|\varphi_{1}-\varphi_{2}\right\|<\delta$, we have

$$
\left|F\left(t, s, \varphi_{1}(s)\right)-F\left(t, s, \varphi_{2}(s)\right)\right|<\varepsilon
$$

and therefore obtain

$$
\left|g\left(t, \varphi_{1}\right)-g\left(t, \varphi_{2}\right)\right| \leq \varepsilon \int_{-\infty}^{0} k(t, s) d s \leq \varepsilon \max _{t \in[0, \omega]} b(t)
$$

This proves that $g$ satisfies (A5). Together with the continuity of $t \mapsto g(t, \varphi)$ (for any $\varphi \in \tilde{X}$ ) it also shows that $g(t, \varphi)$ is continuous on $[0, \omega] \times \tilde{X}$.

Fix $r_{0}, R_{0}$ with $0<r_{0}<R_{0}$. Under (h2)-(h3), it is clear that (A6) is satisfied with $b(t)$ defined by (4.3) and any continuous function $h$ such that

$$
\begin{aligned}
& h(u)=\sup \left\{F(t, s, x): t \in[0, \omega], s \in \mathbb{R}^{-}, x \in\left[R_{0}, u\right]\right\} \quad \text { for } \quad u \geq R_{0} \\
& h(u)=\inf \left\{F(t, s, x): t \in[0, \omega], s \in \mathbb{R}^{-}, x \in\left[u, r_{0}\right]\right\} \quad \text { for } \quad u \leq r_{0} .
\end{aligned}
$$

From Theorem 3.1(a), we derive:

Theorem 4.1. Consider (4.1), assume (h1)-(h3) and (A2)-(A4), let b(t) be as in (4.3) and h(u) as in (4.6). Then there exists a positive periodic solution of (4.1) if $L_{0} C_{1}>1$ and $L^{\infty} C_{2}<1$.

Similarly, we can construct $h(u)$ such that (3.5) holds. This shows that, not only the results in Section 3 apply to broad classes of impulsive and non-impulsive models included in the form (4.1), but also that the sufficient conditions in Theorem 3.1 and its corollaries are easy to check.

Recall also that, as observed previously, in many applications the nonlinearity $g(t, \varphi)$ is strictly sublinear at $\infty$, i.e., $L^{\infty}(h)=0$, thus condition (3.6) reduces to $L_{0}(h) C_{1}(b)>1$ - and that, after rescaling $b$ and $h$ as described in Remark 3.5, we may assume that $L_{0}(h)=1$.

To illustrate our results, we further analise two selected families of impulsive DDEs which fall within the scope of (4.1). They are most relevant in mathematical biology and other sciences, as they include many important models. A few concrete classic examples will also be treated.

\subsection{Integro-differential equations with infinite distributed delay}

The examples treated in [15] can be inserted in the following class of integro-differential equations:

$$
x^{\prime}(t)=-a(t) x(t)+b(t) \int_{-\infty}^{0} k(s) F(t, x(t+s)) d s, \quad t \geq 0,
$$

with $a, b: \mathbb{R}^{+} \rightarrow \mathbb{R}^{+}$continuous and $\omega$-periodic, $k: \mathbb{R}^{-} \rightarrow \mathbb{R}^{+}$integrable and normalized so that $\int_{-\infty}^{0} k(s) d s=1, F: \mathbb{R}^{+} \times \mathbb{R}^{+} \rightarrow \mathbb{R}^{+}$continuous and $\omega$-periodic in the first variable, and $a, b, F$ not identically zero. This equation has the form (4.4), it satisfies (h1)-(h3), and the nonlinearity in (4.5) reads as $g(t, \varphi)=b(t) \int_{-\infty}^{0} k(s) F(t, \varphi(s)) d s$. Next, we consider the impulsive version of (4.7). 
Theorem 4.2. Consider the system

$$
\left\{\begin{array}{l}
x^{\prime}(t)=-a(t) x(t)+b(t) \int_{-\infty}^{0} k(s) F(t, x(t+s)) d s, \quad t \geq 0, t \neq t_{k}, k \in \mathbb{N}, \\
x\left(t_{k}^{+}\right)-x\left(t_{k}\right)=I_{k}\left(x\left(t_{k}\right)\right), \quad k \in \mathbb{N},
\end{array}\right.
$$

assume that $a, b, k, F$ satisfy the above properties as in (4.7), and that conditions (A2)-(A4) are satisfied. For $C_{i}=C_{i}(b), i \in\{1,2,3,4\}$, as in (3.7), (3.9) and $F_{0}, F^{0}, F_{\infty}, F^{\infty} \in[0, \infty]$ defined by

$$
\begin{array}{cc}
F_{0}=\liminf _{u \rightarrow 0^{+}}\left(\min _{t \in[0, \omega]} \frac{F(t, u)}{u}\right), & F^{0}=\limsup _{u \rightarrow 0^{+}}\left(\max _{t \in[0, \omega]} \frac{F(t, u)}{u}\right), \\
F_{\infty}=\liminf _{u \rightarrow \infty}\left(\min _{t \in[0, \omega]} \frac{F(t, u)}{u}\right), & F^{\infty}=\limsup _{u \rightarrow \infty}\left(\max _{t \in[0, \omega]} \frac{F(t, u)}{u}\right),
\end{array}
$$

assume also that either

$$
C_{2} F^{\infty}<1<C_{1} F_{0}
$$

or

$$
C_{3} F_{\infty}>1>C_{4} F^{0}
$$

Then (4.8) has at least one positive $\omega$-periodic solution.

Proof. Assume that $C_{2} F^{\infty}<1<C_{1} F_{0}$. For any $r_{0}, R_{0}$ with e.g. $0<r_{0}<1<R_{0}$, (A6) holds with $b(t)$ the coefficient in (4.8) and $h_{r_{0}, R_{0}}: \mathbb{R}^{+} \rightarrow \mathbb{R}^{+}$a continuous function defined in $\left[0, r_{0}\right] \cup\left[R_{0}, \infty\right)$ by (4.6):

$$
h_{r_{0}, R_{0}}(u)= \begin{cases}\min _{t \in[0, \omega], x \in\left[u, r_{0}\right]} F(t, x) \quad \text { if } \quad 0 \leq u \leq r_{0} . \\ \max _{t \in[0, \omega], x \in\left[R_{0}, u\right]} F(t, x) \quad \text { if } \quad u \geq R_{0} .\end{cases}
$$

For $L_{0}\left(h_{r_{0}, R_{0}}\right)$ as in $(3.3)$, we have $L_{0}\left(h_{r_{0}, R_{0}}\right) \leq F_{0}$ for any $r_{0} \in(0,1)$. If $F_{0}=\infty$, it is clear that $L_{0}\left(h_{r_{0}, R_{0}}\right) \rightarrow \infty$ as $r_{0} \rightarrow 0^{+}$. Otherwise, simple calculations show that, for any $\varepsilon>0$, we may choose $r_{0}$ small enough such that $L_{0}\left(h_{r_{0}, R_{0}}\right) \geq F_{0}-\varepsilon$. In this way, if $1<C_{1}\left(F_{0}-\varepsilon\right)$ the assertion $C_{1} L_{0}\left(h_{r_{0}, R_{0}}\right)>1$ holds true for $r_{0}$ small. An analogous procedure allows us to conclude that we can choose $R_{0}>1$ sufficiently large such that $C_{2} L^{\infty}\left(h_{r_{0}, R_{0}}\right)<1$. The superlinear case is treated in a similar way. Theorem 3.1 implies the result.

For the non-impulsive version (4.7), Corollary 3.3 and Remark 3.3 yield the criterion below.

Corollary 4.1. For (4.7) with $a, b, k, F$ as above, assume $F^{\infty}=0$ and

(a) either $b(t) \geq a(t)$ with $b(t) \not \equiv a(t)$ in $[0, \omega]$ and $F_{0} \geq 1$; (b) or $F_{0} \int_{0}^{\omega} b(t) d t \geq \mathrm{e}^{A(\omega)}-1$.

Then, (4.7) has at least one positive $\omega$-periodic solution.

Remark 4.1. Theorem 3.2 and Corollary 4.1 generalise the work of Jiang and Wei [15], where the authors obtained two results about existence of a positive $\omega$-periodic solution for (4.7): in [15, Theorem 2.1] under the assumptions $F_{0}=\infty, F^{\infty}=0$, and in [15, Theorem 2.2] assuming that $F_{0} \geq 1, F^{\infty}=0$, and $b(t)>a(t)$ for $t \in[0, \omega]$, these latter hypotheses slightly stronger than the requisites in Corollary 4.1(a). 
Straightforward generalisations of these results are derived if (4.7) is replaced by

$$
x^{\prime}(t)=-a(t) x(t)+\sum_{i=1}^{m} b_{i}(t) \int_{-\infty}^{0} k_{i}(s) F_{i}(t, x(t+s)) d s,
$$

where $b_{i}, k_{i}, F_{i}(1 \leq i \leq m)$ are as $b, k, F$ in (4.7). We now give some concrete examples.

Example 4.1. Consider an impulsive equation with distributed delay of Mackey-Glass type:

$$
\left\{\begin{array}{l}
x^{\prime}(t)=-a(t) x(t)+b(t) \int_{-\infty}^{0} k(s) \frac{x(t+s)}{1+c(t) x(t+s)^{n}} d s, \quad t \geq 0, t \neq t_{k}, k \in \mathbb{N}, \\
x\left(t_{k}^{+}\right)-x\left(t_{k}\right)=I_{k}\left(x\left(t_{k}\right)\right), \quad k \in \mathbb{N},
\end{array}\right.
$$

where $a, b, c: \mathbb{R}^{+} \rightarrow \mathbb{R}^{+}$are continuous and $\omega$-periodic, with $a(t) \not \equiv 0, b(t) \not \equiv 0$ and $c(t)>0$ for $t \in[0, \omega], k: \mathbb{R}^{-} \rightarrow \mathbb{R}^{+}$is integrable with $\int_{-\infty}^{0} k(s) d s=1$ and $n>0$. Suppose also that the instants $t_{k}$ and the functions $I_{k}(k \in \mathbb{N})$ satisfy (A2)-(A4). Eq. (4.11) is a particular case of (4.8) with

$$
F(t, x)=\frac{x}{1+c(t) x^{n}} .
$$

With the previous notations, we obtain $F_{0}=1, F^{\infty}=0$ in (4.9). For the constants defined in (3.13) and (3.15), from Corollary 3.1 we have that (4.11) admits at least one positive $\omega$-periodic solution if one of the following conditions is satisfied: (a) $b(t) \geq m_{1} a(t)$ for $t \in[0, \omega]$ with $\max _{t \in[0, \omega]}(b(t)-$ $\left.m_{1} a(t)\right)>0$, and $m_{1} C_{1}^{*} \geq 1$; (b) $C_{1}^{* *} \geq 1$. Thus, for (4.11) with no impulses, Corollary 4.1 asserts that a positive $\omega$-periodic solution must exist if either $\max _{t \in[0, \omega]}(b(t)-a(t))>0, \min _{t \in[0, \omega]}(b(t)-a(t)) \geq 0$, or $\int_{0}^{\omega} b(t) d t \geq \mathrm{e}^{A(\omega)}-1$.

For the sake of illustration, suppose now that $a, b: \mathbb{R}^{+} \rightarrow(0, \infty)$ with $m_{1}=\min _{t \in[0, \omega]} \frac{b(t)}{a(t)}$ and $b\left(t_{0}\right)>m_{1} a\left(t_{0}\right)$ for some $t_{0}$, and first assume in addition that all the impulses are nonpositive, i.e., $I_{k}(u) \leq 0$ for $u \geq 0$ and $k=1, \ldots, p$. Then we have $J_{k}(0)^{s} \geq J_{k}(0)^{i} \geq 1$ for all $k, B_{0} \geq 1=\underline{B}(0)$ and

$$
C_{1}^{*}=\left(\mathrm{e}^{A(\omega)} B^{0}-1\right)^{-1}\left(\mathrm{e}^{A(\omega)}-1\right), \quad C_{1}^{* *}=\left(\mathrm{e}^{A(\omega)} B^{0}-1\right)^{-1} \int_{0}^{\omega} b(s) d s .
$$

Therefore, from Corollary 3.1, the existence of a positive $\omega$-periodic solution of (4.11) follows if

$$
\max \left\{m_{1}\left(\mathrm{e}^{A(\omega)}-1\right), \int_{0}^{\omega} b(s) d s\right\} \geq \mathrm{e}^{A(\omega)} B^{0}-1 .
$$

On the contrary, if all the impulses are nonnegative, i.e., $I_{k}(u) \geq 0$ for $u \geq 0$ and $k=1, \ldots, p$, then $J_{k}(0)^{i} \leq J_{k}(0)^{s} \leq 1$ for all $k, B_{0}=\underline{B}(0)$, and

$$
C_{1}^{*}=\left(\mathrm{e}^{A(\omega)} B^{0}-1\right)^{-1} B_{0}\left(\mathrm{e}^{A(\omega)}-1\right), \quad C_{1}^{* *}=\left(\mathrm{e}^{A(\omega)} B^{0}-1\right)^{-1} B_{0} \int_{0}^{\omega} b(s) d s .
$$

Thus, since $B^{0} \leq 1$, Corollary 3.1 guarantees that (4.11) admits a positive $\omega$-periodic solution if

$$
\max \left\{m_{1},\left(\mathrm{e}^{A(\omega)}-1\right)^{-1} \int_{0}^{\omega} b(s) d s\right\} \geq B_{0}^{-1} .
$$


Recently, there has been an increasing interest in DDEs with mixed monotonicity, where the nonlinear terms involve one or more functions with different delays e.g. of the form $f(t, x(t-\tau(t)), x(t-$ $\sigma(t)))$, with $f(t, x, y)$ monotone increasing in the variable $x$ and monotone decreasing in $y$. Although small delays are in general harmless, in the sense that the delayed model has the same global properties of the equation without delays, the presence of two or more delays in the same nonlinear function may change this situation drastically, as illustrated in [3]. However, DDEs with different delays in the same nonlinear term appear naturally in real-world models, see $[17,32]$ and references therein. The global dynamics of such equations have been the subject of a few recent studies, where questions of stability, persistence, permanence, existence of periodic solutions were addressed [1, 3, 4, 9, 12, 32]. Nevertheless, as far as the authors know, only the case of discrete delays has been considered.

The next example is a model with mixed monotonicity, where the different delays in the nonlinear terms have a different nature: discrete and distributed. Two further comments are in order. First, this example shows that the choice of $\sigma$ for the cone $K=K_{\sigma}$ may have an important role. Secondly, to work on $K$ is very useful to treat DDEs with a mixed monotonicity, since the definition of $K$ provides natural uniform lower and upper bounds for functions $y \in K: \sigma\|y\| \leq y(t) \leq\|y\|$ on $[0, \omega]$.

Example 4.2. Consider an impulsive Nicholson-type equation given by

$$
\left\{\begin{array}{l}
x^{\prime}(t)=-a(t) x(t)+\sum_{i=1}^{m} b_{i}(t) x\left(t-\sigma_{i}(t)\right) \int_{-\infty}^{0} k_{i}(s) \mathrm{e}^{-c_{i}(t) x(t+s)} d s, t \geq 0, t \neq t_{k}, \\
x\left(t_{k}^{+}\right)-x\left(t_{k}\right)=I_{k}\left(x\left(t_{k}\right)\right), \quad k \in \mathbb{N} .
\end{array}\right.
$$

where $a, b_{i}, c_{i}, \sigma_{i}: \mathbb{R}^{+} \rightarrow \mathbb{R}^{+}$are continuous and $\omega$-periodic, with $a(t) \not \equiv 0, b(t):=\sum_{i=1}^{m} b_{i}(t) \not \equiv 0$ and $c_{i}(t)>0$ for $t \in[0, \omega], k_{i}: \mathbb{R}^{-} \rightarrow \mathbb{R}^{+}$are integrable with $\int_{-\infty}^{0} k_{i}(s) d s=1, i=1, \ldots, m$, and $t_{k}, I_{k}(u)(k \in \mathbb{N})$ satisfy (A2),(A3),(A4). Here, $g$ in (4.5) reads as

$$
g(t, \varphi)=\sum_{i=1}^{m} f_{i}\left(t, \varphi\left(-\sigma_{i}(t)\right), \varphi\right), \quad t \geq 0, \varphi \in \tilde{X},
$$

where $f_{i}(t, x, \varphi):=b_{i}(t) x \int_{-\infty}^{0} k_{i}(s) e^{-c_{i}(t) \varphi(s)} d s$ increases in the argument $x \in \mathbb{R}^{+}$and decreases in the argument $\varphi \in \tilde{X}^{+}, i=1, \ldots, m$. In the next result, we explore the criteria in Corollary 3.1.

Proposition 4.1. Fix $K=K_{\sigma}$, where $\sigma=(\underline{B} / \bar{B}) \mathrm{e}^{-A(\omega)}$ as in Lemma 2.2. Under the above conditions and notations, (4.12) admits at least one positive $\omega$-periodic solution if in addition one of the following statements holds:

(a) $C_{1}^{*} \sum_{i=1}^{m} b_{i}(t) \geq \frac{\bar{B}}{\underline{B}} a(t) \mathrm{e}^{A(\omega)}$ on $[0, \omega]$ and $C_{1}^{*} \sum_{i=1}^{m} b_{i}\left(t_{0}\right)>\frac{\bar{B}}{\underline{B}} a\left(t_{0}\right) \mathrm{e}^{A(\omega)}$ for some $t_{0}$;

(b) $C_{1}^{* *} \geq \frac{\bar{B}}{B} \mathrm{e}^{A(\omega)}$

In particular, the non-impulsive version of (4.12) has a positive $\omega$-periodic solution if either $\gamma(t):=$ $\sum_{i=1}^{m} b_{i}(t)-a(t) e^{A(\omega)} \geq 0$ on $[0, \omega]$ and $\gamma(t) \not \equiv 0$, or $\sum_{i=1}^{m} \int_{0}^{\omega} b_{i}(t) d t \geq \mathrm{e}^{A(\omega)}\left(\mathrm{e}^{A(\omega)}-1\right)$.

Proof. We adapt the reasoning in the former example. Write $b(t)=\sum_{i=1}^{m} b_{i}(t)$. Define $\underline{c_{i}}=$ $\min _{[0, \omega]} c_{i}(t), \overline{c_{i}}=\max _{[0, \omega]} c_{i}(t), \underline{c}=\min _{1 \leq i \leq m} \underline{c_{i}}, \bar{c}=\max _{1 \leq i \leq m} \overline{c_{i}}$. Recall that the function $\theta(\bar{u})=$ $u \mathrm{e}^{-c u}$, where $c>0$, attaints its maximum $(c \overline{\mathrm{e}})^{-1}$ at $u=c^{-1}$ and is increasing on $\left[0, c^{-1}\right]$. Take $r_{0}, R_{0}>0$, with $r_{0}<\bar{c}^{-1}$ and $\sigma R_{0}>\underline{c}^{-1}$. 
Let $t \geq 0$, and $y \in K$, and note that $y(t) \geq \sigma\|y\|$. If $R_{0} \leq y \leq R$, it holds

$$
g\left(t, y_{t}\right) \leq \sum_{i=1}^{m} b_{i}(t) y\left(t-\sigma_{i}(t)\right) \mathrm{e}^{-\underline{c} \sigma\|y\|} \leq b(t)\|y\| \mathrm{e}^{-\underline{c} \sigma\|y\|} \leq b(t)(\underline{c} \sigma \mathrm{e})^{-1} .
$$

And if $r \leq y \leq r_{0}$, we have

$$
g\left(t, y_{t}\right) \geq \sum_{i=1}^{m} b_{i}(t) y\left(t-\sigma_{i}(t)\right) \mathrm{e}^{-\bar{c}\|y\|} \geq b(t) \sigma\|y\| \mathrm{e}^{-\bar{c}\|y\|} \geq b(t) \sigma r \mathrm{e}^{-\bar{c} r} .
$$

Thus (3.4) is satisfied with $h: \mathbb{R}^{+} \rightarrow \mathbb{R}^{+}$continuous such that $h(R)=(\underline{c} \sigma \mathrm{e})^{-1}$ for $R \geq R_{0}$ and $h(r)=\sigma r \mathrm{e}^{-\bar{c} r}$ for $r \leq r_{0}$, for which $L_{0}=\sigma$ and $L^{\infty}=0$. If (a) is satisfied, then take $m_{1}=\left(C_{1}^{*} \sigma\right)^{-1}$; the result follows from Corollary 3.1(a) and Remark 3.3. If (b) holds, we apply Corollary 3.1(b).

We remark that Chen [4] considered the DDE

$$
y^{\prime}(t)+a(t) y(t)=b(t) y(t-\sigma(t)) \mathrm{e}^{-c(t) y(t-\tau(t)))},
$$

with $a(t), b(t), \sigma(t), \tau(t), c(t)$ positive, $\omega$-periodic continuous functions, and used the continuation theorem of degree theory to prove the existence of a positive periodic solution provided that $\int_{0}^{\omega} b(s) d s>$ $A(\omega) \mathrm{e}^{2 A(\omega)}$; this condition is more restrictive than $\int_{0}^{\omega} b(s) d s \geq \mathrm{e}^{A(\omega)}\left(\mathrm{e}^{A(\omega)}-1\right)$ in Proposition 4.1.

\subsection{Integro-differential equations with periodic distributed delay}

Consider the family of impulsive DDEs

$$
\left\{\begin{array}{l}
x^{\prime}(t)=-a(t) x(t)+\beta(t) \int_{t-\tau(t)}^{t} c(s) H(s, x(s)) d s, \quad t \geq 0, t \neq t_{k}, k \in \mathbb{N} \\
x\left(t_{k}^{+}\right)-x\left(t_{k}\right)=I_{k}\left(x\left(t_{k}\right)\right), \quad k \in \mathbb{N}
\end{array}\right.
$$

with the functions $a, \beta, \tau: \mathbb{R}^{+} \rightarrow \mathbb{R}^{+}, c: \mathbb{R} \rightarrow \mathbb{R}^{+}, H: \mathbb{R} \times \mathbb{R}^{+} \rightarrow \mathbb{R}^{+}$continuous, $\omega$-periodic in $t$ and not identically zero. Assume also (A2)-(A4). See e.g. [2,6] for non-impulsive versions of (4.13). This system has the form (4.1), with

$$
k(t, s)=\beta(t) c(s+t) \chi_{[-\tau(t), 0]}(s), \quad F(t, s, x)=H(s+t, x),
$$

so that the nonlinearity is given by $g(t, \varphi)=\beta(t) \int_{-\tau(t)}^{0} c(s+t) H(s+t, \varphi(s)) d s$. Of course, (h1)-(h3) are fulfilled. Set

$$
b(t)=\beta(t) \int_{-\tau(t)}^{0} c(s+t) d s .
$$

(A6) is satisfied with $h: \mathbb{R}^{+} \rightarrow \mathbb{R}^{+}$continuous if we define $h(u)$ as in (4.6) on $\left[0, r_{0}\right] \cup\left[R_{0}, \infty\right)$, for $0<r_{0}<R_{0}$, thus the results for the sublinear case in Section 3 can be applied. 
Example 4.3. System (4.13) represents a periodic Nicholson's blowfly model with periodic distributed finite delay $\tau(t)$ when

$$
F(t, s, x)=H(s+t, x)=x \mathrm{e}^{-d(t+s) x},
$$

where $d: \mathbb{R} \rightarrow(0, \infty)$ is a continuous $\omega$-periodic function, in which case we obtain the system

$$
\left\{\begin{array}{l}
x^{\prime}(t)=-a(t) x(t)+\beta(t) \int_{t-\tau(t)}^{t} c(s) x(s) \mathrm{e}^{-d(s) x(s)} d s, \quad t \geq 0, t \neq t_{k}, k \in \mathbb{N} \\
x\left(t_{k}^{+}\right)-x\left(t_{k}\right)=I_{k}\left(x\left(t_{k}\right)\right), \quad k \in \mathbb{N}
\end{array}\right.
$$

Following along the lines in Example 4.2, for $\bar{d}$ and $\underline{d}$ the maximum and minimum values of $d$, respectively, choose $r_{0}=\bar{d}^{-1}$ and $R_{0}=\underline{d}^{-1}$, so that in (4.6) we obtain $h(u)=u \mathrm{e}^{-\bar{d} u}$ if $0 \leq u<r_{0}$, $h(u)=R_{0} \mathrm{e}^{-\underline{d} R_{0}}$ if $u \geq R_{0}$, for which $L_{0}(h)=1, L^{\infty}(h)=0$. Under the stated conditions on $a, \beta, \tau, c, d$ and (A2)-(A4), Theorem 4.1 asserts that (4.15) has at least one positive $\omega$-periodic solution if $C_{1}(b)>1$. In particular, the non-impulsive Nicholson equation

$$
x^{\prime}(t)=-a(t) x(t)+\beta(t) \int_{t-\tau(t)}^{t} c(s) x(s) \mathrm{e}^{-d(s) x(s)} d s, \quad t \geq 0,
$$

admits at least one positive $\omega$-periodic solution if either

$$
\max _{t \in[0, \omega]}\left(\beta(t) \int_{t-\tau(t)}^{t} c(s) d s-a(t)\right)>0, \min _{t \in[0, \omega]}\left(\beta(t) \int_{t-\tau(t)}^{t} c(s) d s-a(t)\right) \geq 0
$$

or

$$
\int_{0}^{\omega} \beta(t) \int_{t-\tau(t)}^{t} c(s) d s d t \geq \mathrm{e}^{\int_{0}^{\omega} a(t) d t}-1 .
$$

In [2], Amster and Idels proved this result for (4.16) under the sufficient condition

$$
\min _{t \in[0, \omega]} c(t)>\max _{t \in[0, \omega]} \frac{a(t)}{\beta(t) \tau(t)},
$$

which is much more restrictive than (4.17). In summary, we improve significantly the criterion in [2].

If instead of $H(t, x)=x \mathrm{e}^{-d(t) x}$ in (4.13) we choose $H(t, x)=x^{\gamma} \mathrm{e}^{-d(t) x}$ where $\gamma \in(0,1)$, we obtain a modified version of the Nicholson model, for which there are $b(t), h(u)$ such that (3.4) is true and $L_{0}(h)=\infty, L^{\infty}(h)=0$. Theorem 3.1 guarantees that there exists a positive $\omega$-periodic solution, regardless the sign or profile of the impulses, provided that they verify (A2)-(A4). If one chooses $H(t, x)=\frac{x}{1+d(t) x^{n}}(n>0)$ in (4.13), a Mackey-Glass-type model with distributed periodic delay is obtained; conclusions as the ones for (4.15) can be stated.

\subsection{Effect of the impulses}

It turns out that the introduction of impulses in periodic scalar DDEs can create positive period solutions, which do not exist otherwise. For instance, in [5, 9] concrete examples of a periodic DDE of the form (1.3) exhibiting a positive period solution were given, for which the zero solution of the associated equation without impulses is a global attractor of all its positive solutions. Consequently, 
it is worth while to illustrate circumstances under which the impulses satisfy our set of assumptions, and moreover either generate or at least do not destroy periodic orbits.

Thus, we go back to impulsive equations (1.1), suppose that $a(t), g(t, \varphi)$ in (4.5) satisfy (A1), (A5) and (A6) with, as often occurs in applications (see the above examples), functions $b(t), h(u)$ such that $L_{0}=1, L^{\infty}=0$. Within this scenario, we now give examples of impulsive functions $I_{k}(u)$ whose sign may vary, for which (A2)-(A4) and condition $C_{1}>1$ are fulfilled, ensuring that a positive $\omega$-period solution must exist.

Example 4.4. Take $I_{k}(u)=I(u):=\sin (u)$ for all $k$, and suppose that $2^{p}<\mathrm{e}^{A(\omega)}$. Then (A3), (A4) are satisfied with $a_{k}=-1 / \pi, b_{k}=1$ and $J(0):=J_{k}(0)^{s}=J_{k}(0)^{i}=2^{-1}$, for all $k \in \mathbb{N}$. Moreover, $C_{1}=C_{1}(b)>1$ holds if $\min _{t \in[0, \omega]} \int_{t}^{t+\omega} b(s) \mathrm{e}^{\int_{t}^{s} a(u) d u} d s>\mathrm{e}^{A(\omega)}-2^{p}$. In particular, $C_{1}>1$ if $\int_{0}^{\omega} b(s) d s \geq \mathrm{e}^{A(\omega)}-2^{p}$. Thus, impulses can create a periodic solution.

To illustrate this situation, consider a periodic DDE with multiple discrete delays and subject to the above impulses:

$$
\left\{\begin{array}{lr}
x^{\prime}(t)=-a(t) x(t)+\sum_{i=1}^{m} f_{i}\left(t, x\left(t-\tau_{i}(t)\right)\right), & 0 \leq t \neq t_{k}, \\
x\left(t_{k}^{+}\right)-x\left(t_{k}\right)=\sin \left(x\left(t_{k}\right)\right), & k \in \mathbb{N},
\end{array}\right.
$$

with $a(t)>0, \tau_{i}(t) \geq 0, f_{i}(t, u) \geq 0$ continuous, w-periodic in $t, f_{i}(t, u)$ bounded $(1 \leq i \leq m)$ and such that

$$
b(t) h^{-}(u) \leq \sum_{i=1}^{m} f_{i}(t, u) \leq b(t) h^{+}(u) \quad \text { for } \quad t \in[0, \omega], u \geq 0,
$$

for some continuous functions $b, h^{-}, h^{+}: \mathbb{R}^{+} \rightarrow \mathbb{R}^{+}$satisfying

$$
\begin{aligned}
& h^{+}(u)<u \quad \text { for } \quad u>0, \\
& h^{ \pm}(0)=0, \quad\left(h^{ \pm}\right)^{\prime}(0)=1 .
\end{aligned}
$$

For the non-impulsive equation $x^{\prime}(t)=-a(t) x(t)+\sum_{i=1}^{m} f_{i}(t, x(t-\tau(t)))$, Theorem 3.2 in [8] implies that its zero solution is globally asymptotically stable (in the set of all nonnegative solutions) if $b(t) \leq a(t), t \in[0, \omega]$. On the other hand, (A6) holds. Thus, if there exists $p \in \mathbb{N}$ such that

$$
\mathrm{e}^{A(\omega)}-\int_{0}^{\omega} b(s) d s \leq 2^{p}<\mathrm{e}^{A(\omega)}
$$

the introduction of $p$ impulses on each interval of length $\omega$ generates at least one positive $\omega$-periodic solution for (4.20); e.g. with $\int_{0}^{\omega} a(s) d s=2 \int_{0}^{\omega} b(s) d s=1$, it suffices to implement one impulse $\Delta\left(x\left(t_{1}\right)\right)=\sin \left(x\left(t_{1}\right)\right)$, for any $t_{1} \in\left[0, \omega\left[\right.\right.$, an repeat it at times $t_{1}+k \omega, k \in \mathbb{N}$. Note also that the requirements $(4.21),(4.22)$ are satisfied by a broad family of equations, which includes Nicholson and Mackey-Glass type equations.

Example 4.5. Let $p=2 m$ with $m \in \mathbb{N}, \beta \in(0,1)$ and $\delta>0$. Define $I_{k}(u)=(-1)^{k} \beta u \mathrm{e}^{-\delta u}$ for $k \in \mathbb{N}$ and $u \geq 0$. Hence, $\left|I_{k}(u)\right| / u<\beta$, there exist $J_{k}(0):=\lim _{u \rightarrow 0^{+}} J_{k}(u)=\left(1+(-1)^{k} \beta\right)^{-1}$ for all $u>0$ and $k \in \mathbb{N}$. Here, $a_{k}=-\beta, b_{k}=0$ for $k$ odd and $a_{k}=0, b_{k}=\beta$ for $k$ even, and (A2)-(A4) are satisfied if $(1+\beta)^{m}<\mathrm{e}^{A(\omega)}$. We have $C_{1}>1$ if

$$
\min _{t \in[0, \omega]} \int_{t}^{t+\omega} b(s) \mathrm{e}^{\int_{t}^{s} a(u) d u} \prod_{k: t_{k} \in[t, s)} J_{k}(0) d s>\frac{\mathrm{e}^{A(\omega)}}{\left(1-\beta^{2}\right)^{m}}-1 .
$$


In particular, this condition is fulfilled if $\int_{0}^{\omega} b(s) d s \geq(1+\beta)\left(\frac{\mathrm{e}^{A(\omega)}}{\left(1-\beta^{2}\right)^{m}}-1\right)$.

Example 4.6. Define the impulse functions as $I_{k}(u)=I(u):=\beta u \mathrm{e}^{-\delta u}-\alpha u$, for any $k \in \mathbb{N}$, where $\beta>0>\alpha-1$ and $\delta>0$. Note that, for $u>0$, the impulses are always negative if $\beta \leq \alpha$, always positive if $\alpha<0$, whereas the sign of the impulses changes if $\beta>\alpha>0$, with $I^{\prime}(0)=\beta-\alpha>0$ and $I(\infty)=-\infty$. With the previous notation, $a_{k}=-\alpha, b_{k}=\beta-\alpha, J_{k}(u)=\left(1+\beta \mathrm{e}^{-\delta u}-\alpha\right)^{-1}$, hence

$$
\begin{aligned}
& J(0):=\lim _{u \rightarrow 0^{+}} J_{k}(u)=(1+\beta-\alpha)^{-1}, \quad J(\infty):=\lim _{u \rightarrow \infty} J_{k}(u)=(1-\alpha)^{-1}, \quad k \in \mathbb{N}, \\
& B_{0}=B^{0}=(1+\beta-\alpha)^{-p} .
\end{aligned}
$$

Hypotheses (A2)-(A4) are fulfilled if $(1+\beta-\alpha)^{p}<\mathrm{e}^{A(\omega)}$. Here, $C_{1}>1$ holds if

$$
\min _{t \in[0, \omega]} \int_{t}^{t+\omega} b(s) \mathrm{e}^{\int_{t}^{s} a(u) d u} \prod_{k: t_{k} \in[t, s)}(1+\beta-\alpha)^{-1} d s>\left(\mathrm{e}^{A(\omega)}(1+\beta-\alpha)^{-p}-1\right) .
$$

In particular, $J(0) \geq 1$ if $\beta \leq \alpha$, and we get $C_{1}>1$ provided that $\int_{0}^{\omega} b(s) d s \geq \mathrm{e}^{A(\omega)}(1+\beta-\alpha)^{-p}-1$. For $\beta>\alpha, J(0)<1$; thus, $C_{1}>1$ if either $b(t) \geq a(t), t \in[0, \omega]$, or $\int_{0}^{\omega} b(s) d s \geq \mathrm{e}^{A(\omega)}-(1+\beta-\alpha)^{p}$.

\section{$5 \quad$ Final comments}

In this paper, we apply a Krasnoselskii-Guo fixed point theorem to establish sufficient conditions under which the impulsive periodic DDE (1.1) admits at least one positive periodic solution. The novelty of our approach derives from the operator $\Phi$ constructed in (2.3), whose fixed points are the periodic solutions we are looking for. Our method allows the treatment of equations with a very general nonlinearity $g$ and infinite delay, subject to impulses whose signs may vary. Applications to Volterra integro-differential equations (4.1) are given. In particular, the study of a Nicholsontype equation with mixed monotonicity is included as an illustration of our results. Eq. (1.1) is broad enough to incorporate as special cases a large number of problems studied by many authors, nevertheless our criteria strongly improve several known results.

The present paper pursues the study in the former work of Faria and Oliveira [9], which dealt with Eq. (1.2). The main result here, Theorem 3.1, generalises [9, Theorem 2.3] in several ways: (i) as mentioned above, infinite delays as well as nonlinear impulses are allowed in (1.1); (ii) rather than being bounded, $g$ is now allowed to have sublinear growth at infinity; the superlinear case is also addressed; (iii) finally, following the suggestion in [9, Remark 2.1], here limits inferior and superior are used in (3.6) and (3.8), instead of limits.

Although several open problems posed in [9] were solved here, relevant lines of future investigation were not addressed. Namely, the global attractivity of a positive periodic solution to (1.1) was not studied: it depends heavily on the particular shape of the nonlinearity $g$, and more conditions on the impulses should be imposed. Also, it would be desirable to adapt the present approach to quasiperiodic DDEs, due to their relevance in real world phenomena. On the other hand, our method can be extended to other classes of impulsive scalar DDEs, as outlined briefly in Remark 3.2. The ideas developed here can be carefully adjusted to deal with some classes of periodic impulsive $n$-dimensional systems, as it will be shown in a forthcoming paper. 


\section{Acknowledgements}

This work was supported by Ministerio de Ciencia, Innovación y Universidades of Spain under grant FPU16/04416 (Sebastián Buedo-Fernández) and by Fundação para a Ciência e a Tecnologia under project UID/MAT/04561/2019 (Teresa Faria). This work was partially written while S. BuedoFernández was visiting the research center CMAF-CIO of the University of Lisbon, and he thanks the university for its kind hospitality.

\section{References}

[1] Amster P, Balderrama R. Existence and multiplicity of periodic solutions for a generalised hematopoiesis model. J. Appl. Math. Comput. 2017; 55(1-2):591-607.

[2] Amster P, Idels L. Periodic solutions in general scalar non-autonomous models with delays. Nonlinear Differential Equations Appl. 2013; 20(5):1577-1596.

[3] Berezansky L, Braverman E. A note on stability of Mackey-Glass equations with two delays. J. Math. Anal. Appl. 2017; 450(2):1208-1228.

[4] Chen Y. Periodic solutions of delayed periodic Nicholson's blowflies models. Can. Appl. Math. Q. 2003; 11(1):23-28.

[5] Dai B, Bao L. Positive periodic solutions generated by impulses for the delay Nicholson's blowflies model. Electron. J. Qual. Theory Differ. Equ. 2016; 2016(4):1-11.

[6] Faria T. Periodic solutions for a non-monotone family of delayed differential equations with applications to Nicholson systems. J. Differential Equations 2017; 263(1):509-533.

[7] Faria T, Gadotti MC, Oliveira JJ. Stability results for impulsive functional differential equations with infinite delay. Nonlinear Anal. 2012; 75(18):6570-6587.

[8] Faria T, Obaya R, Sanz AM. Asymptotic behaviour for a class of non-monotone delay differential systems with applications. J. Dyn. Diff. Equ. 2018; 30(3): 911-935.

[9] Faria T, Oliveira JJ. Existence of positive periodic solutions for scalar delay differential equations with and without impulses J. Dyn. Diff. Equ. https://doi.org/10.1007/s10884-017-9616-0.

[10] Graef J, Kong LJ. Existence of multiple periodic solutions for first order functional differential equations. Math. Comput. Model. 2011; 54 (11-12):2962-2968.

[11] Guo DJ, Lakshmikantham V. Nonlinear Problems in Abstract Cones. New York: Academic Press; 1988.

[12] Györi I, Hartung F, Mohamady NA. Permanence in a class of delay differential equations with mixed monotonicity, Electron. J. Qual. Theory Differ. Equ. 2018; 2018(53):1-21.

[13] Hale JK, Kato J. Phase space for retarded equations with infinite delay. Funkcial. Ekvac. 1978; 21(1):1141.

[14] Hino Y, Murakami S, Naito T. Functional Differential Equations with Infinite Delay. New-York: SpringerVerlag; 1993. 
[15] Jiang D, Wei J. Existence of positive periodic solutions for Volterra integro-differential equations. Acta Math. Sci. Ser. B. 2001; 21(4):553-560.

[16] Krasnoselskii MA. Positive Solutions of Operator Equations. Groningen: P. Noordhoff, Ltd.; 1964.

[17] Langlois GP, Craig M, Humphries AR et al. Normal and pathological dynamics of platelets in humans. J. Math. Biol. 2017; 75(6-7):1411-1462

[18] Li J, Du C. Existence of positive periodic solutions for a generalized Nicholson's blowflies model. J. Comput. Appl. Math. 2008; 221(1): 226-233.

[19] Li X, Lin X, Jiang D, Zhang X. Existence and multiplicity of positive periodic solutions to functional differential equations with impulse effects. Nonlinear Anal. 2005; 62(4):683-701.

[20] Liu X, Takeuchi Y. Periodicity and global dynamics of an impulsive delay Lasota-Wazewska model. J. Math. Anal. Appl. 2007; 327(1):326-341.

[21] Meng Q, Yan J. Existence and n-multiplicity of positive periodic solutions for impulsive functional differential equations with two parameters. Bound. Value Probl. 2015; 2015(212):1-10.

[22] Nieto JJ. Basic theory for nonresonance impulsive periodic problems of first order. J. Math. Anal. Appl. 1997; 205(2):423-433.

[23] Nieto JJ, Uzal JM. Pulse positive periodic solutions for some classes of singular nonlinearities. Appl. Math. Lett. 2018; 86:134-140.

[24] Ouahab A. Existence and uniqueness results for impulsive functional differential equations with scalar multiple delay and infinite delay. Nonlinear Anal. 2007; 67(4):1027-1041.

[25] Samoilenko AM, Perestyuk NA. Impulsive Differential Equations. Singapore: World Scientific; 1995.

[26] Tang XH, Zou X. The existence and global exponential stability of a periodic solution of a class of delay differential equations. Nonlinearity. 2009; 22(10):2423-2442.

[27] Wan A, Jiang D, Xu X. A new existence theory for positive periodic solutions to functional differential equations. Comput. Math. Applic. 2004; 47(8-9):1257-1262.

[28] Yan J. Existence and global attractivity of positive periodic solution for an impulsive Lasota-Wazewska model. J. Math. Anal. Appl. 2003; 279(1):111-120.

[29] Yan J. Stability for impulsive delay differential equations. Nonlinear Anal. 2005; 63(1):66-80.

[30] Yan J. Existence of positive periodic solutions of impulsive functional differential equations with two parameters. J. Math. Anal. Appl. 2007; 327(2):854-868.

[31] Yan J, Zhao A, Nieto JJ. Existence and global attractivity of positive periodic solution of periodic singlespecies impulsive Lotka-Volterra systems. Math. Comput. Modelling. 2004; 40(5-6): 509-518.

[32] Yuan Y, Bélair J. Threshold dynamics in an SEIRS model with latency and temporary immunity. J. Math. Biol. 2014; 69(4):875-904.

[33] Zhang R, Huang Y, Wei T. Positive periodic solution for Nicholson-type delay systems with impulsive effects. Adv. Difference Equ. 2015; 2015(371):1-16. 
[34] X. Zhang, M. Feng. Multi-parameter, impulsive effects and positive periodic solutions of first-order functional differential equations. Bound. Value Probl. 2015; 2015(137):1-22.

[35] Zhang X, Yan J, Zhao A. Existence of positive periodic solutions for an impulsive differential equation. Nonlinear Anal. 2008; 68(10):3209-3216.

[36] Zhao XQ. Permanence implies the existence of interior periodic solutions for FDEs. Internat. J. Qualitative Theory Differential Equations Appl. 2008; 2(1):125-137. 